\title{
Nicotinamide Riboside and
} Metformin Ameliorate Mitophagy Defect in Induced Pluripotent Stem Cell-Derived Astrocytes With POLG Mutations

OPEN ACCESS

Edited by:

Andreas Hermann,

University Hospital of Rostock,

Germany

Reviewed by:

Anne Grünewald,

University of Luxembourg,

Luxembourg

Nataliia Naumova,

University of Padua, Italy

*Correspondence:

Kristina Xiao Liang

Xiao.Liang@uib.no

Laurence A. Bindoff

Laurence.Bindoff@uib.no

Xingang $L$

lixg@sdu.edu.cn

tThese authors have contributed equally to this work and share last

authorship

Specialty section:

This article was submitted to

Stem Cell Research,

a section of the journal

Frontiers in Cell and Developmental

Biology

Received: 06 July 2021

Accepted: 27 August 2021

Published: 24 September 2021

Citation:

Chen A, Kristiansen CK, Hong Y, Kianian A, Fang EF, Sullivan GJ,

Wang J, Li X, Bindoff LA and Liang KX (2021) Nicotinamide

Riboside and Metformin Ameliorate Mitophagy Defect in Induced Pluripotent Stem Cell-Derived

Astrocytes With POLG Mutations. Front. Cell Dev. Biol. 9:737304. doi: 10.3389/fcell.2021.737304
Anbin Chen 1,2,3,4, Cecilie Katrin Kristiansen ${ }^{3,4}$, Yu Hong ${ }^{3,4}$, Atefeh Kianian ${ }^{3}$, Evandro Fei Fang ${ }^{5,6}$, Gareth John Sullivan ${ }^{7,8,9,10}$, Jian Wang ${ }^{1,2,11}$, Xingang Li ${ }^{1,2 *}$, Laurence A. Bindoff ${ }^{3 * 4}$ and Kristina Xiao Liang ${ }^{3,4 * t}$

${ }^{1}$ Department of Neurosurgery, Qilu Hospital and Institute of Brain and Brain-Inspired Science, Cheeloo College of Medicine, Shandong University, Jinan, China, ${ }^{2}$ Shandong Key Laboratory of Brain Function Remodeling, Jinan, China, ${ }^{3}$ Department of Clinical Medicine (K1), University of Bergen, Bergen, Norway, ${ }^{4}$ Neuro-SysMed, Center of Excellence for Clinical Research in Neurological Diseases, Department of Neurology, Haukeland University Hospital, Bergen, Norway, ${ }^{5}$ Department of Clinical Molecular Biology, Akershus University Hospital, University of Oslo, Oslo, Norway, ${ }^{6}$ The Norwegian Centre on Healthy Ageing, Oslo, Norway, ${ }^{7}$ Department of Molecular Medicine, Institute of Basic Medical Sciences, University of Oslo, Oslo, Norway, ${ }^{8}$ Institute of Immunology, Oslo University Hospital, Oslo, Norway, ${ }^{9}$ Hybrid Technology Hub - Centre of Excellence, Institute of Basic Medical Sciences, University of Oslo, Oslo, Norway, ${ }^{10}$ Department of Pediatric Research, Oslo University Hospital, Oslo, Norway, ${ }^{11}$ Department of Biomedicine, University of Bergen, Bergen, Norway

Mitophagy specifically recognizes and removes damaged or superfluous mitochondria to maintain mitochondrial homeostasis and proper neuronal function. Defective mitophagy and the resulting accumulation of damaged mitochondria occur in several neurodegenerative diseases. Previously, we showed mitochondrial dysfunction in astrocytes with POLG mutations, and here, we examined how POLG mutations affect mitophagy in astrocytes and how this can be ameliorated pharmacologically. Using induced pluripotent stem cell (iPSC)-derived astrocytes carrying POLG mutations, we found downregulation of mitophagy/autophagy-related genes using RNA sequencingbased KEGG metabolic pathway analysis. We confirmed a deficit in mitochondrial autophagosome formation under exogenous stress conditions and downregulation of the mitophagy receptor p62, reduced lipidation of LC3B-II, and decreased expression of lysosome protein lysosomal-associated membrane protein 2A (LAMP2A). These changes were regulated by the PINK1/Parkin pathway and AKT/mTOR/AMPK/ULK1 signaling pathways. Importantly, we found that double treatment with nicotinamide riboside (NR) and metformin rescued mitophagy defects and mitochondrial dysfunction in POLG-mutant astrocytes. Our findings reveal that impaired mitophagy is involved in the observed mitochondrial dysfunction caused by POLG mutations in astrocytes, potentially contributing to the phenotype in POLG-related diseases. This study also demonstrates the therapeutic potential of NR and metformin in these incurable mitochondrial diseases.

Keywords: mitophagy, astrocytes, POLG, IPSC (induced pluripotent stem cells), nicotinamide riboside (NR), metformin, mitochondria 


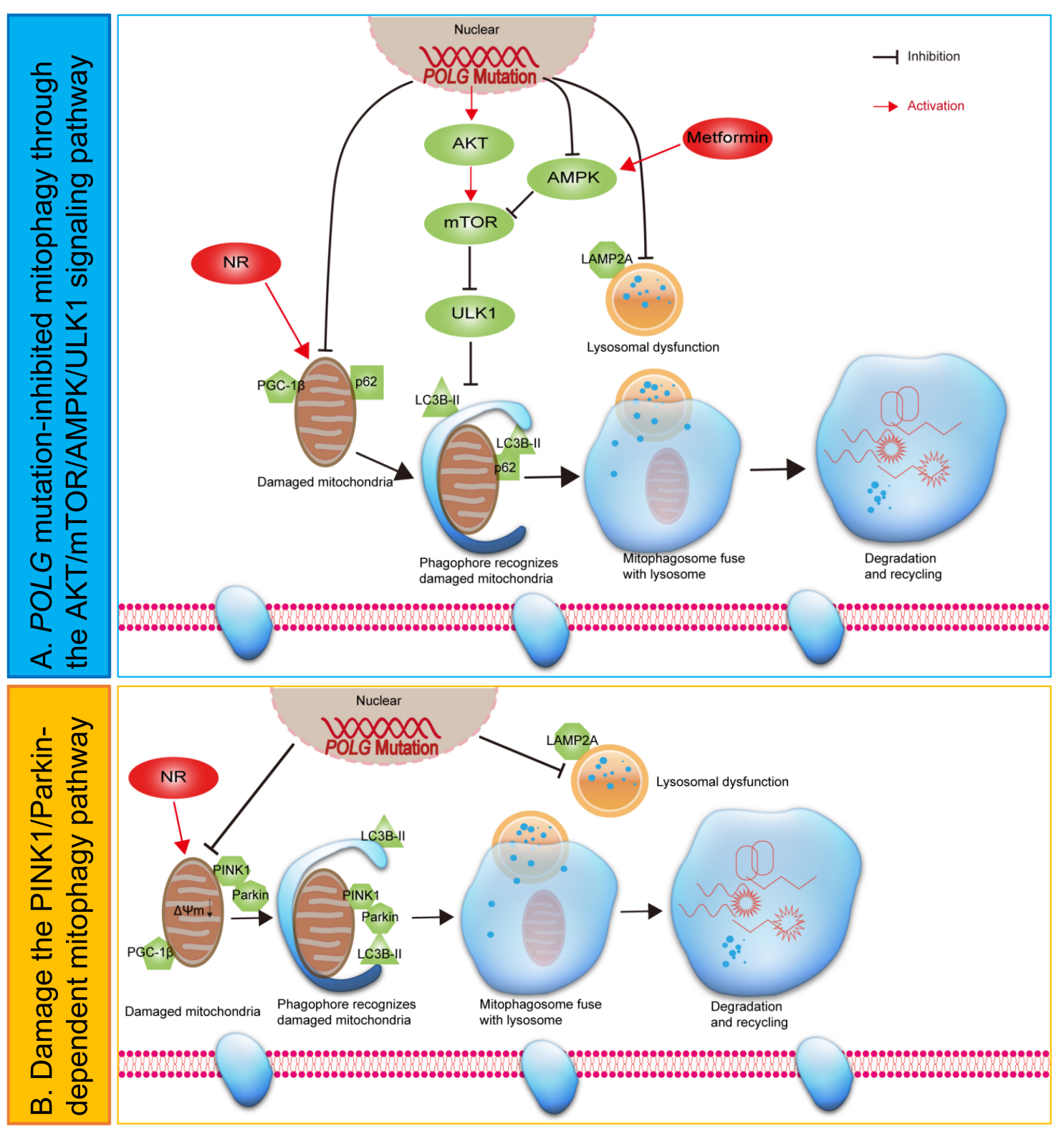

GRAPHICAL ABSTRACT | Hypothetical mechanisms of mitophagy dysfunction in POLG-mutant astrocytes.

\section{INTRODUCTION}

Mitochondrial DNA (mtDNA) polymerase $\gamma(\operatorname{pol} \gamma)$ replicates the mitochondrial genome and the holoenzyme consists of a catalytic subunit (encoded by $P O L G$ ) and a dimeric form of its accessory subunit (encoded by POLG2) (Graziewicz et al., 2006). Mutations in $P O L G$ are associated with a wide range of mitochondrial diseases that form a continuum from catastrophic early-onset hepato-cerebral degeneration to lateonset progressive external ophthalmoplegia (Hikmat et al., 2020).

Mitochondria are fundamental, subcellular organelles with multiple functions including ATP generation and participating in the regulation of cell metabolism, calcium $\left(\mathrm{Ca}^{+}\right)$signaling, redox state, neurotransmission, and plasticity (Lin and Beal, 2006; Mattson et al., 2008; Sheng and Cai, 2012; Fang et al., 2016; Kerr et al., 2017). Mutations in POLG lead to neuronal loss conditioned by abnormal mtDNA homeostasis comprising both depletion and a time-dependent increase in mtDNA damage, along with loss of complex I (Tzoulis et al., 2014). We have demonstrated that these findings could be replicated using induced pluripotent stem cell (iPSC)-derived neural stem cells (NSCs) in which we also found evidence of increased reactive oxygen species (ROS) production and cellular senescence (Liang et al., 2020b). In iPSC-derived neurons, we also showed mitochondrial dysfunction and mtDNA depletion in dopaminergic neurons from POLG patients (Liang et al., 2021). 


\section{A}

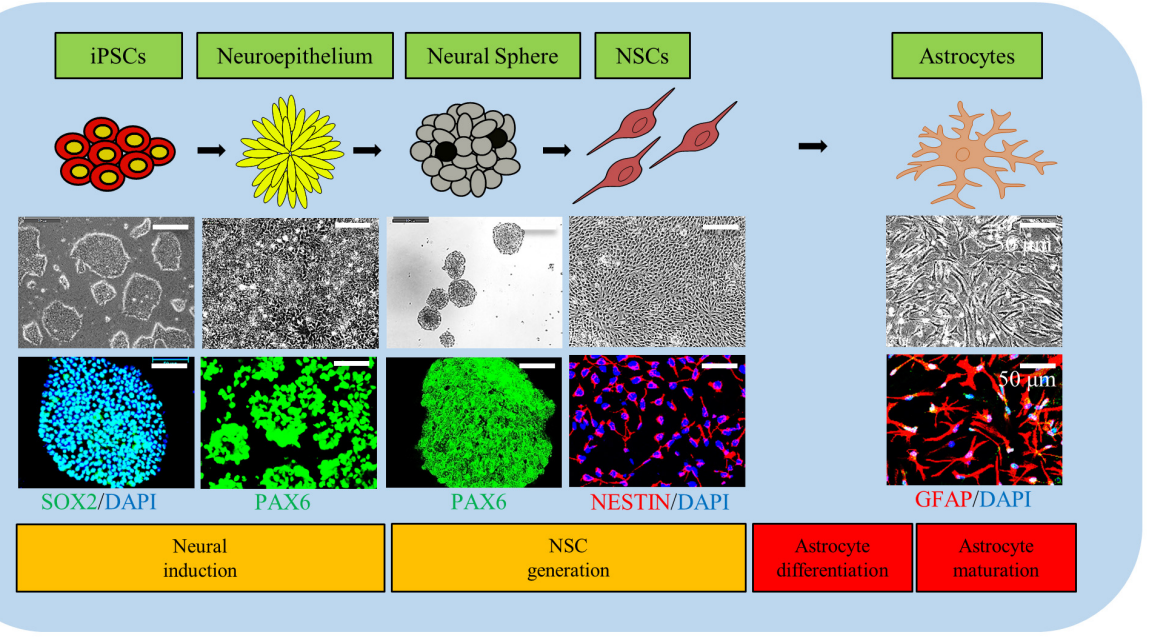

B

GFAP

DAPI

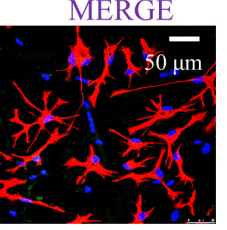

C

EAAT-1

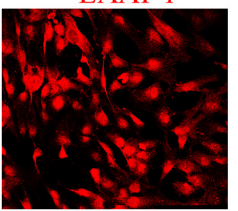

GS
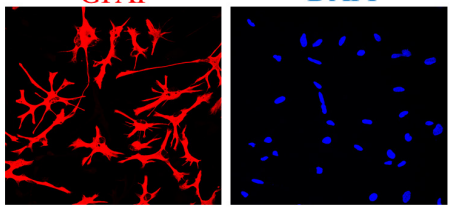

DAPI
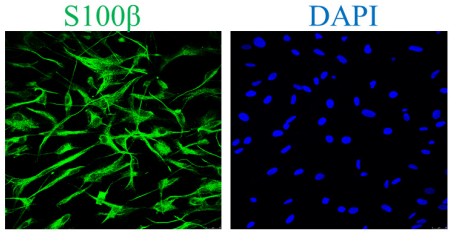

MERGE

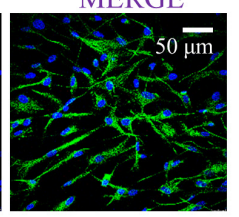

DAPI
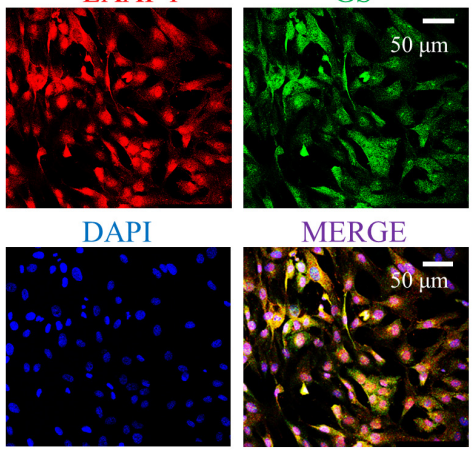

MERGE
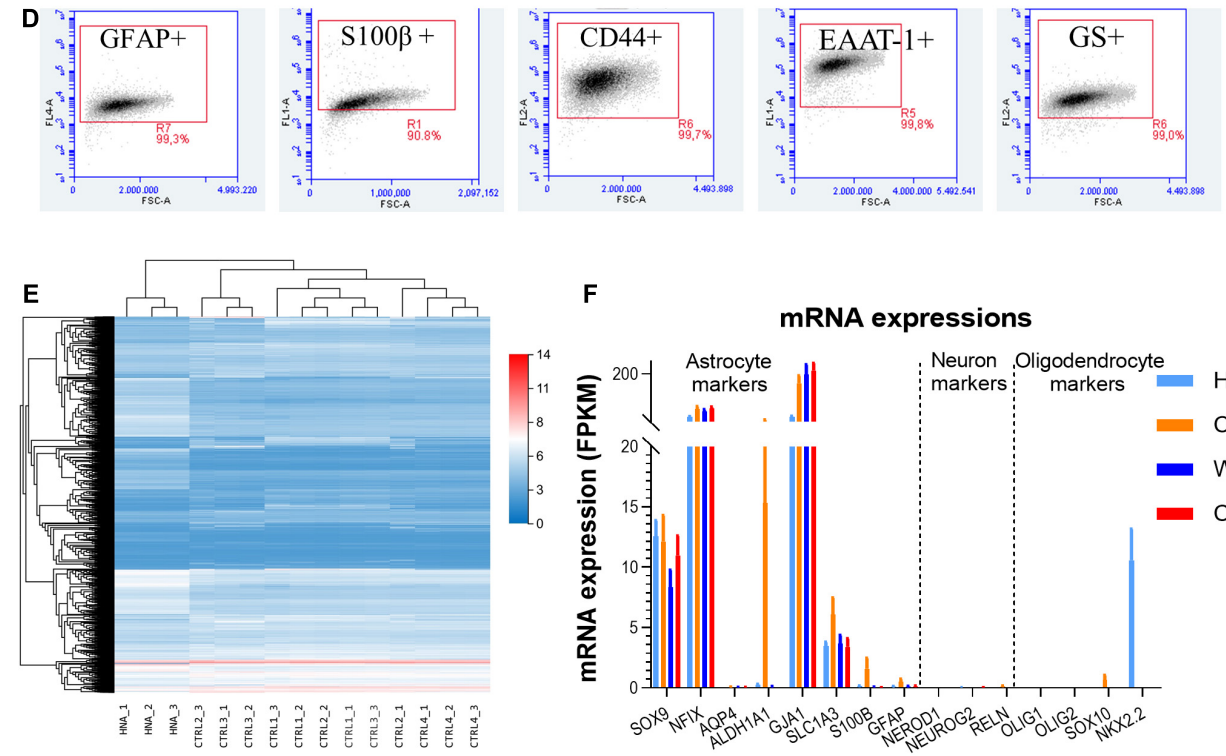

F

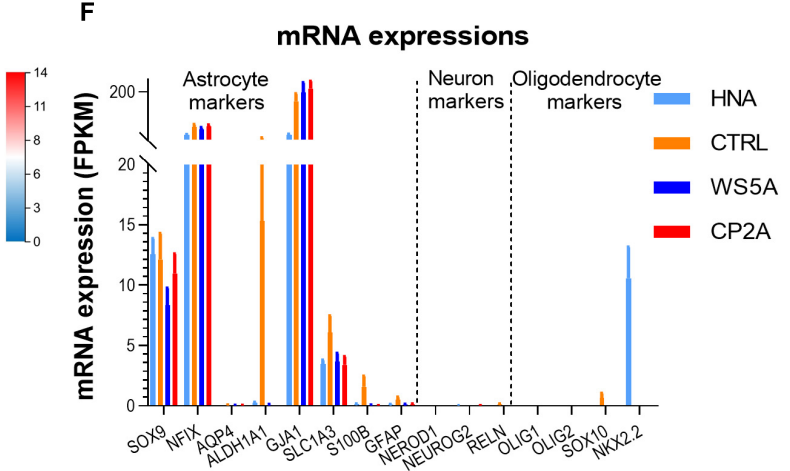

FIGURE 1 | Generation and characterization of patient-specific astrocytes carrying POLG mutations. (A) Schematic of dual differentiation and seeding paradigm for astrocytes (upper panel), representative phase-contrast images (middle panel) and immunostaining for specific stages during neural induction from iPSCs to astrocytes (lower panel). (B,C) Representative phase-contrast images and confocal images of immunostaining for GFAP (red), S100ß (green), EAAT-1 (red), and GS (green). Nuclei are stained with DAPI (blue). Scale bar is $50 \mu \mathrm{m}$. (D) Flow cytometric analysis for percentage of the positive cells expressing GFAP, S100ß, CD44, EAAT-1, and GS. (E) Clustering heat map [log (value + 1)] for DEGs in HNA and control iPSC-derived astrocytes. (F) mRNA expression of astrocyte, neuron, and oligodendrocyte-related genes in HNA and all iPSC-derived astrocytes. The different markers are listed on the $x$-axis, and mRNA expression is on the $y$-axis. Results are expressed as fragments per kilobase million (FPKM). Data information: The data in B-D represent one clone from AG05836 control. The data in $\mathbf{E}$ represent HNA, four different control astrocytes with three clones from Detroit 551 control and one clone from AG05836 control. The data in $\mathbf{F}$ represent HNA, four different control astrocytes with three clones from Detroit 551 control and one clone from AG05836 control, three clones from WS5A and two clones from CP2A. 
Mitochondria are dynamic organelles, and mitochondrial fusion and fission are essential processes for maintaining homeostasis via the segregation of damaged mitochondria (Burté et al., 2015). Fusion and fission are also essential for mitophagy, the selective autophagy pathway that recognizes and degrades damaged or superfluous mitochondria (Youle and Narendra, 2011; Fivenson et al., 2017; Lautrup et al., 2019). Accumulating empirical evidence suggests that mitophagy impairment contributes to neurodegenerative diseases such as Alzheimer's disease (AD) and Parkinson's disease (PD) (Kerr et al., 2017; Fang et al., 2019b). Several mitophagy pathways are recognized including the PINK1/Parkin pathway (Vives-Bauza et al., 2010; Deas et al., 2011) and the energysensitive PI3K/AKT/mTOR/ULK1 axis (Egan et al., 2011; Hung et al., 2021). The kinase mammalian target of rapamycin (mTOR) is a major negative regulator of mitophagy/autophagy (Heras-Sandoval et al., 2014), and AMP-activated protein kinase (AMPK) is recognized as an important mitophagy activator, acting through a pleiotropic mechanism involving mTOR inhibition and direct phosphorylation/activation of Unc-51-like kinase 1 (ULK1) (Kim et al., 2011; Hung et al., 2021). The mTOR is a downstream target of both phosphatidylinositol 3 kinase (PI3K), and AKT and is activated by receptors of both neurotrophic and growth factors, promoting cell growth, differentiation, and survival (Manning and Toker, 2017).

Astrocytes are the major glial cell type in the central nervous system and serve multiple functions including synaptic signaling, neurotransmitter synthesis and recycling, as well as nutrient uptake and regulation of local blood flow (Haydon and Carmignoto, 2006). Many of these processes depend on local metabolism and/or energy utilization. Astrocytes respond to increases in neuronal activity and metabolic demand by upregulating glycolysis, but also possess a significant capacity for mitochondrial energy metabolism (Jackson and Robinson, 2018). Growing evidence also links astrocyte dysfunction with neurodegenerative disease (Acosta et al., 2017; Liddelow et al., 2017; Richetin et al., 2020; Sonninen et al., 2020). While astrocytic mitophagy/autophagy plays a fundamental role in maintaining brain function and health, disease-driven astrocytic mitophagy/autophagy impairment can potentiate inflammation, oxidative stress, and neural death (Maldonado et al., 2018; Wang and $\mathrm{Xu}, 2020$ ).

A previous study in mice links mitochondrial dysfunction in astrocytes with POLG-related disease (Ignatenko et al., 2018). In an earlier study, we showed that POLG-mutant astrocytes exhibited mitochondrial dysfunction including loss of mitochondrial membrane potential, loss of ATP production, loss of complex I, disturbed $\mathrm{NAD}^{+} / \mathrm{NADH}$ metabolism, and mtDNA depletion (Liang et al., 2020a). However, whether and how POLG mutations affect mitophagy in these cells remains unclear. In this study, we aimed to identify how mitochondrial dysfunction driven by POLG mutations impacts mitophagy. We used astrocytes derived from iPSCs of two POLG patients, one homozygous for c.2243G > C; p.W748S and one compound heterozygous for c.1399G > A/c.2243G > C; p.A467T/W748S mutations. We identified a defect in mitophagy with lowered lysosomal activity and abnormal regulation of multiple pathways including PINK1/Parkin and AKT/mTOR/AMPK/ULK1. More importantly, treatment with nicotinamide riboside (NR) and metformin rescued the impaired mitophagy via upregulating the SIRT1/AMPK pathway and downregulating the mTOR pathway, which further improved mitochondrial function. Our results show, for the first time, that $P O L G$ mutations induce mitophagy impairment and that this defect is treatable.

\section{MATERIALS AND METHODS}

\section{Ethics Approval}

The project was approved by the Western Norway Committee for Ethics in Health Research (REK nr. 2012/919).

\section{Generation of Induced Pluripotent Stem Cells, Neural Stem Cells, and Astrocyte Differentiation}

The fibroblasts from one homozygous c.2243G > C, p.W748S/W748S (WS5A) and one compound heterozygous c.1399G > A/c.2243G > C, p.A467T/W748S (CP2A) patient were collected by punch biopsy. Detroit 551 fibroblasts (ATCC ${ }^{\circledR}$ CCL $110^{\mathrm{TM}}$ ) and AG05836B fibroblasts (RRID: CVCL_2B58) were used as control lines. The generation and maintenance of iPSCs and their derived NSCs were described previously (Liang et al., 2020a,b, 2021). Astrocytes were differentiated from NSCs according to the protocol described previously (Liang et al., 2020a). In addition, commercial human normal astrocytes (HNA) (Lonza) was applied as a control. Details of the samples used in this study are described in Supplementary Table 1.

\section{Nicotinamide Riboside and Metformin Treatment}

Nicotinamide riboside was provided by Evandro Fei Fang (University of Oslo, Norway). The cells were seeded in sixwell plates and treated with metformin (Sigma-Aldrich, St. Louis, MO, United States) and/or NR for $72 \mathrm{~h}$. The medium was changed daily.

\section{Immunofluorescence Staining}

Cells were fixed with 4\% (v/v) paraformaldehyde (PFA) and blocked using blocking buffer containing $10 \%(\mathrm{v} / \mathrm{v})$ normal goat serum (Sigma-Aldrich) with $0.3 \%$ (v/v) Triton ${ }^{\mathrm{TM}} \mathrm{X}-100$ (SigmaAldrich). The cells were then incubated with primary antibody solution overnight at $4^{\circ} \mathrm{C}$ and further stained with secondary antibody solution (1:800 in blocking buffer) for $1 \mathrm{~h}$ at room temperature (RT). The coverslips were mounted onto cover slides using ProLong ${ }^{\mathrm{TM}}$ diamond antifade mounting medium with DAPI (Invitrogen, Carlsbad, CA, United States). The detailed information of the antibodies used is provided in Supplementary Tables 2, 3 . 
A

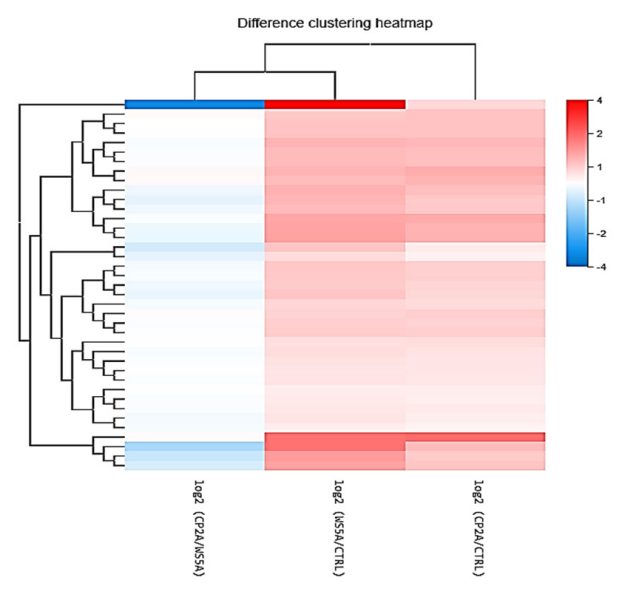

B

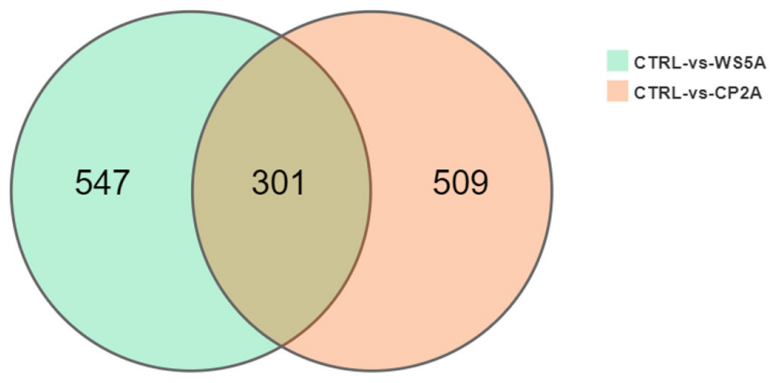

C
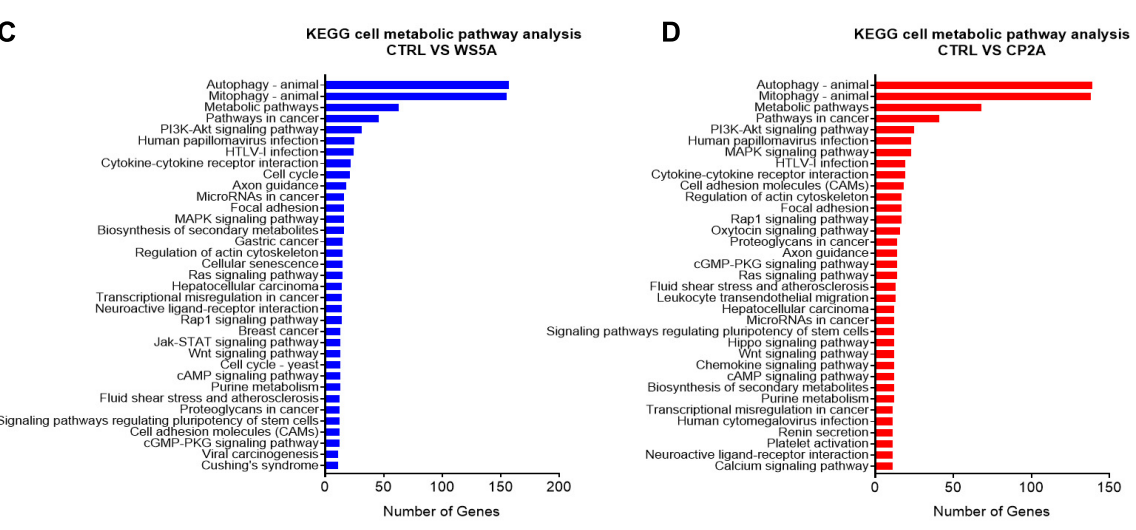

D

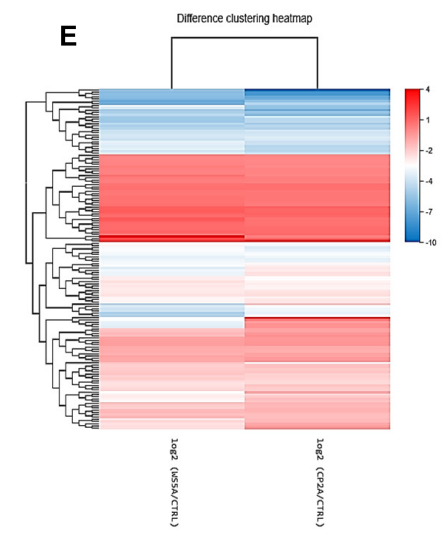

$\mathbf{F}$

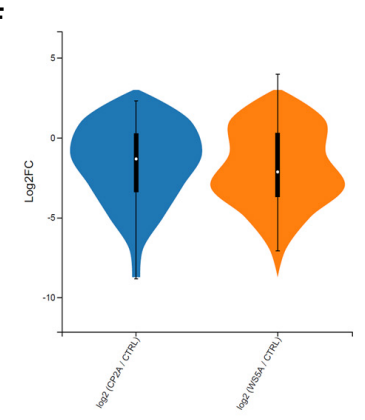

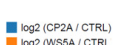
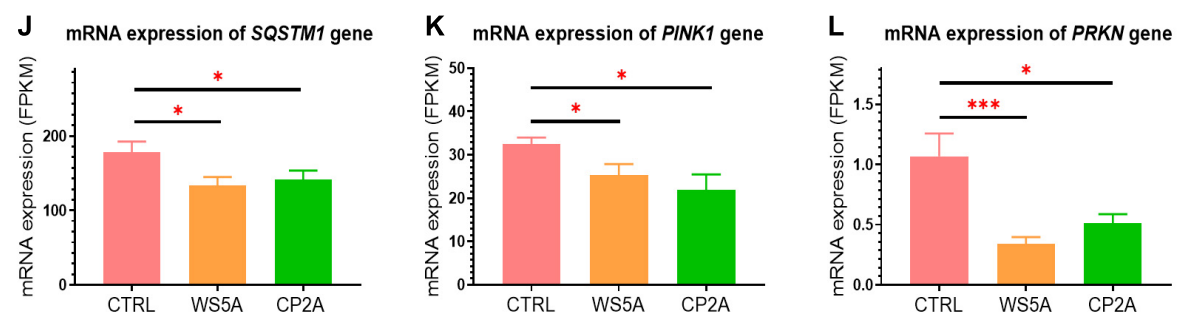

FIGURE 2 | (Continued)
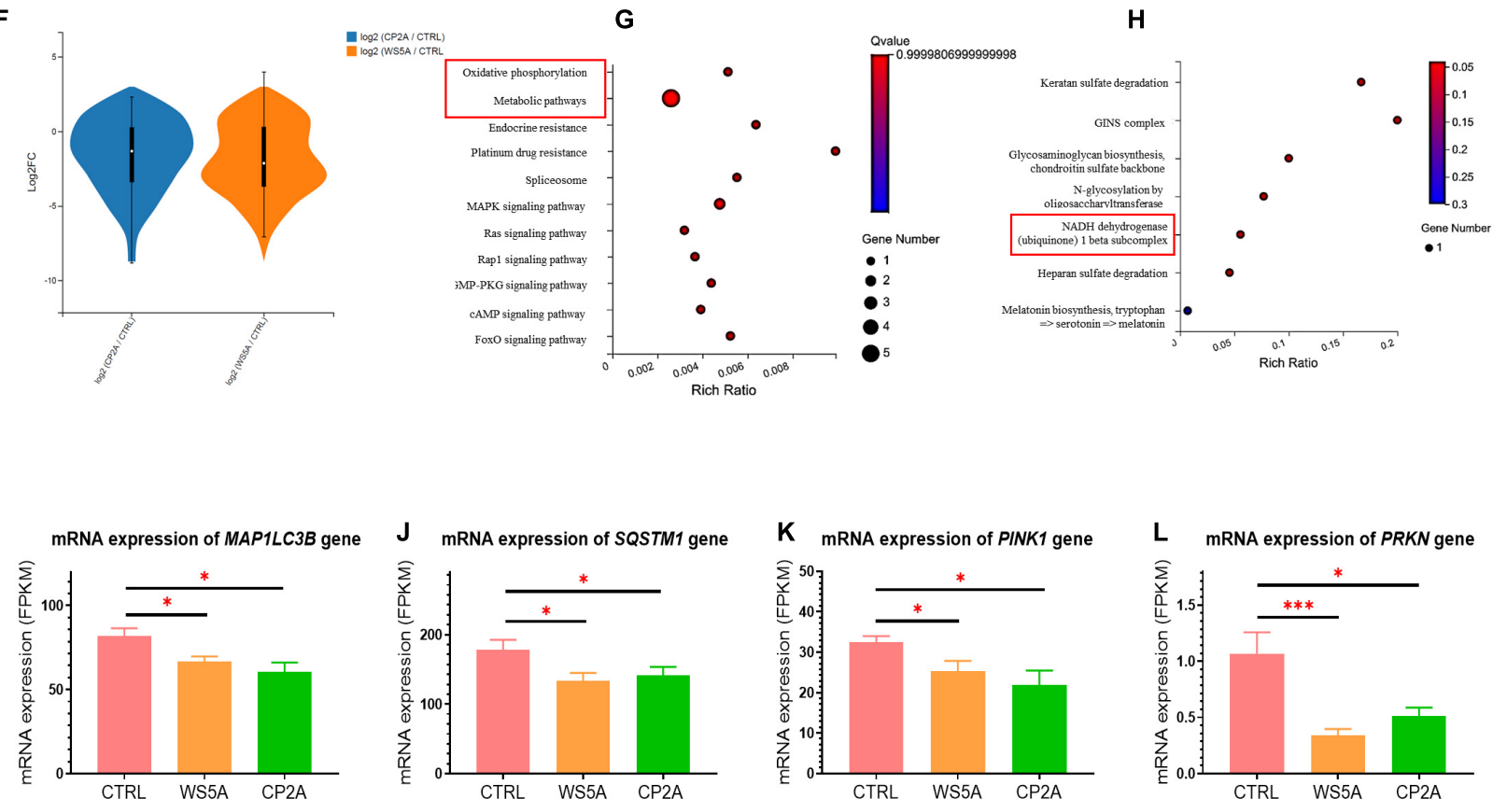
FIGURE 2 | Transcriptomic analysis shows downregulation of mitophagy/autophagy in POLG-mutant astrocytes. (A) Clustering heap map [log (value + 1)] for DEGs in WS5A, CP2A, and control astrocytes. (B) Venn diagram of the DEGs in two compared groups of WS5A astrocytes vs. controls and CP2A astrocytes vs. controls. (C,D) KEGG metabolic pathway analysis for DEGs in two compared groups of WS5A astrocytes vs. controls (C) and CP2A astrocytes vs. controls (D). (E) Unsupervised clustering of DEGs in mitophagy/autophagy pathways in WS5A astrocytes vs. controls and CP2A astrocytes vs. controls. (F) Differential analysis of mitophagy-related genes in patient astrocytes compared to controls. (G) KEGG pathway enrichment analysis of the mitophagy-related genes in patient astrocytes compared to controls. (H) KEGG module enrichment analysis of the mitophagy-related genes in patient astrocytes compared to controls. (I-L) RNA-seq analysis for the mRNA expression of mitophagy-related genes MAP1LC3B (I), SQS1M1 (J), PINK1 (K), and PRKN (L) in WS5A, CP2A, and control astrocytes. Results are expressed as FPKM. Data information: The data in I-L represent four different control astrocytes, including three clones from Detroit 551 control and one clone from AG05836 control, three clones from WS5A and two clones from CP2A. Data are presented as mean \pm SEM for the number of samples ( $n \geq 3$ per clone). Mann-Whitney $U$ test was used for the data presented in $\mathbf{I}-\mathbf{L}$. Significance is denoted for $p$-values of less than 0.05 . ${ }^{*} p<0.05 ;{ }^{* \star \star} p<0.001$.

\section{Live Cell Staining for Mitochondrial Morphology}

Cells were seeded on $\mu$-Slide 4 Well (Ibidi) and cultured at $37^{\circ} \mathrm{C}$ in a humidified incubator overnight. The cells were stained with $500 \mathrm{nM}$ MitoTracker Green (MTG) (Invitrogen) for $45 \mathrm{~min}$ at $37^{\circ} \mathrm{C}$. Images were captured using the Leica TCS SP8 confocal microscope (Leica Microsystems).

\section{Mitochondrial Volume and Membrane Potential Measurement}

Cells were stained with 150 nM MitoTracker Deep Red (MTDR) (Invitrogen) and $150 \mathrm{nM}$ MTG (Invitrogen) for $45 \mathrm{~min}$. Cells treated with $100 \mu \mathrm{M}$ FCCP (Abcam, Cambridge, MA, United States) were used as negative control. Stained cells were detached with TrypLE ${ }^{\mathrm{TM}}$ express (Invitrogen) and analyzed on a FACS BD Accuri ${ }^{\mathrm{TM}}$ C6 flow cytometer (BD Biosciences, San Jose, CA, United States). The data analysis was performed using the Accuri ${ }^{\mathrm{TM}}$ C6 software (BD Biosciences).

\section{Mitophagy Detection Assay}

Astrocytes were seeded on $\mu$-Slide 4 Well and cultured at $37^{\circ} \mathrm{C}$ overnight. The cells were then treated with $10 \mu \mathrm{g} / \mathrm{mL}$ FCCP for $24 \mathrm{~h}$ and were co-stained with mitophagy dye and lysosome dye using a Mitophagy Detection Kit (Dojindo) according to the instruction. MTDR was used to label the mitochondria. Images were captured using the Leica TCS SP8 confocal microscope (Leica Microsystems, Wetzlar, Germany). Quantitative measurements of immunofluorescence images were performed with Image J software (Image J 1.52a; Wayne Rasband National Institutes of Health, United States ${ }^{1}$ ). More than five areas were randomly chosen for quantitative evaluations.

\section{Mitochondrial Complex I Enzyme Activity Assay}

We used the Complex I Enzyme Activity Microplate Assay Kit (Abcam: ab109721) to measure complex I activity. All steps were performed according to the manufacturer's protocol. Cells were seeded in six-well plates at a density of 200,000 cells per well before the initiation of the experiment. Six hours after seeding, cells were treated with different concentrations of metformin for $72 \mathrm{~h}$ and then harvested. Extracted samples were loaded into a 96-well microplate and incubated for $3 \mathrm{~h}$ before washing three times with buffer. Assay solution was added, and the reaction was measured using a microplate reader (INFINITE

${ }^{1}$ https://imagej.nih.gov/ij
F50) with a wavelength of $450 \mathrm{~nm}$ for $90 \mathrm{~min}$. Activity is expressed as the change in absorbance per minute $(\mathrm{mOD} / \mathrm{min})$ per $130 \mu \mathrm{g}$ of cell lysate.

\section{Western Blotting}

Extraction of protein was performed using $1 \times$ RIPA extraction buffer (Thermo Fisher Scientific, Carlsbad, CA, United States) supplemented with protease inhibitor cocktail and phosphatase inhibitor cocktail (Thermo Fisher Scientific). Protein concentration was determined using Pierce ${ }^{\mathrm{TM}}$ BCA Protein Assay Kit (Thermo Fisher Scientific). The cell protein was loaded into NuPAGE ${ }^{\mathrm{TM}} 4-12 \%$ Bis-Tris Protein Gels (Invitrogen) and resolved in the PVDF membrane (BioRad, Hercules, CA, United States) using the Trans-Blot ${ }^{\circledR}$ Turbo $^{\text {TM }}$ transfer system (BioRad). Membranes were blocked with 5\% non-fat dry milk or $5 \%$ bovine serum albumin (BSA) in TBST for $1 \mathrm{~h}$ at RT. Membranes were then incubated overnight at $4^{\circ} \mathrm{C}$ with primary antibodies. After washing in TBST three times, membranes were incubated with donkey anti-mouse antibody or swine anti-rabbit antibody conjugated to horseradish peroxidase secondary for $1 \mathrm{~h}$ at RT. Super Signal West Pico Chemiluminescent Substrate Kit (Thermo Fisher Scientific) was used as enzymesubstrate according to the manufacturer's recommendations. The membranes were visualized by ChemiDoc imaging systems (BioRad). Further details of the used antibodies are provided in Supplementary Tables 2, 3.

\section{RNA Sequencing}

Total RNA was extracted using QIAGEN RNeasy Kit (QIAGEN, Hilden, Germany). Library preparation was conducted at BGI following the guide of the standard protocol. Library preparation (BGISEQ-500RS High-throughput sequencing kit, PE50, V3.0, MGI Tech Co., Ltd., Shenzhen, China), hybridization, and sequencing were performed according to the manufacturer's procedure from BGI (BGI-Shenzhen, China). The sequencing was performed at BGI-Shenzhen using the BGISEQ-500 system. The sequencing data were filtered to remove very low-quality reads using SOAP nuke (v1.5.2) software. The processed FASTQ files were mapped to the human transcriptome and genome using HISAT2 (v2.0.4). The genome version was GRCh38, with annotations from Bowtie2 (v2.2.5). The expression level of the gene was calculated by RSEM (v1.2.12). Differential expression was done with DEseq2 (v1.4.5) package. To take an insight into the change of phenotype, $\mathrm{KEGG}^{2}$ enrichment analysis of annotated differentially expressed genes (DEGs) were performed

\footnotetext{
${ }^{2}$ https://www.kegg.jp
} 
A

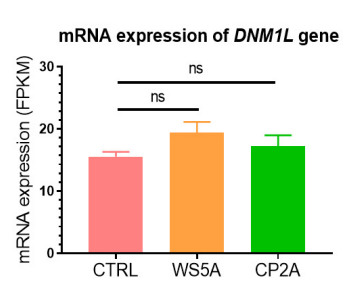

D

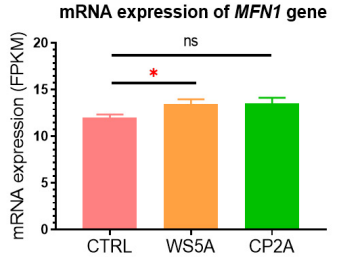

G
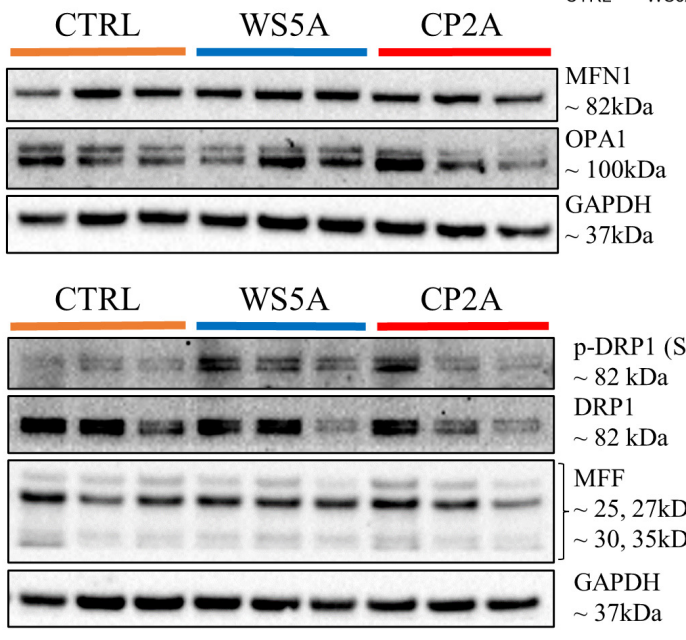

H
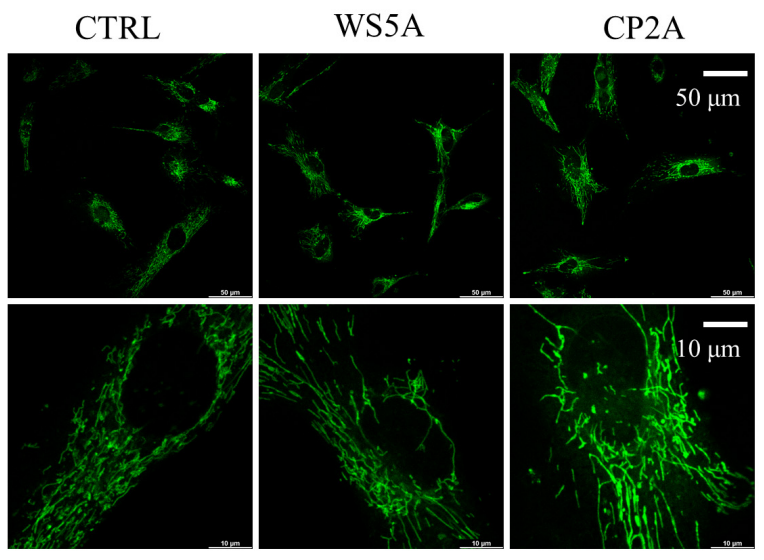

p-DRP1 (Ser 616)

$\sim 82 \mathrm{kDa}$

DRP1

$\sim 82 \mathrm{kDa}$

MFF

$\sim 25,27 \mathrm{kDa}$ $\sim 30,35 \mathrm{kDa}$

GAPDH

$\sim 37 \mathrm{kDa}$
CP2A

WS5A

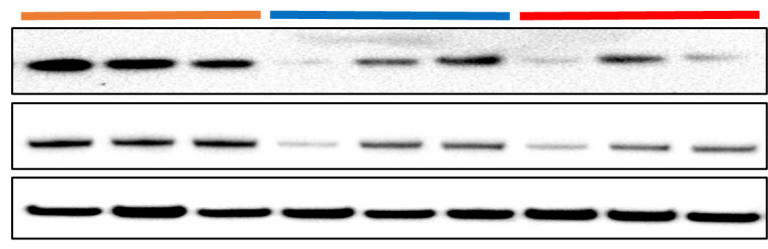

C mRNA expression of MFF gene

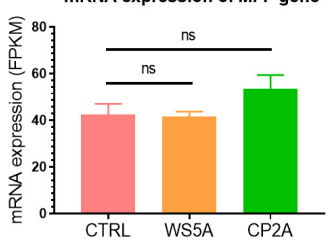

F

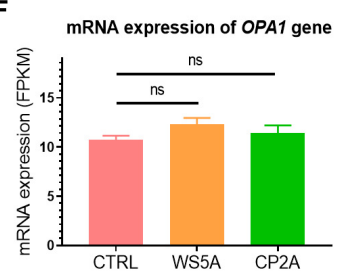

Fusion protein expressions

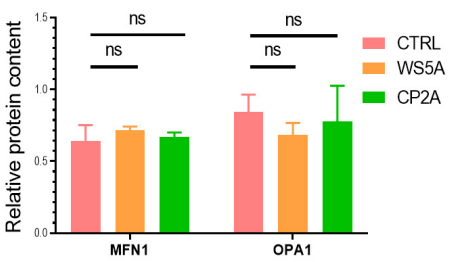

Fission protein expressions

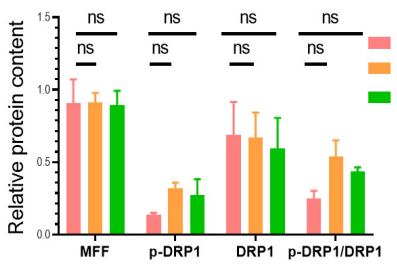

MFF $\quad$ p-DRP1 DRP1 $p$-DRP1/DRP1

Total MTDR

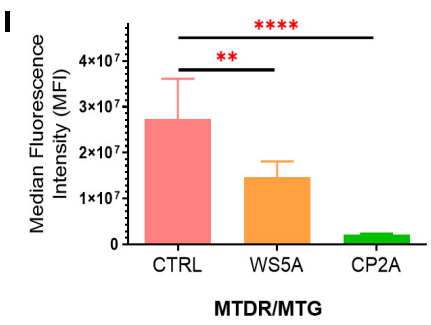

J

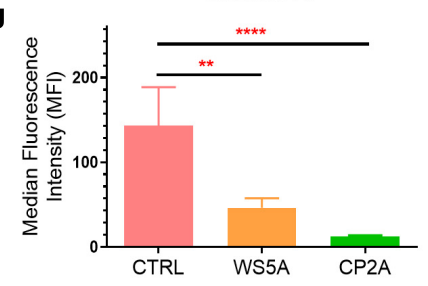

TOMM20 and PGC1- $\beta$ expressions

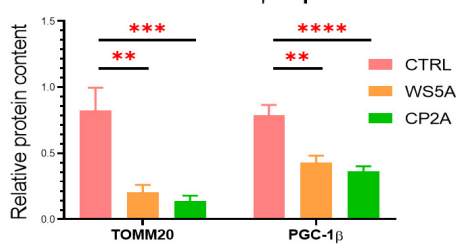

FIGURE 3 | (Continued) 


\begin{abstract}
FIGURE 3 | POLG-mutant astrocytes exhibit loss of mitochondrial volume and impaired mitochondrial function. (A-F) RNA-seq analysis for the mRNA expression of fusion/fission-related genes DNM1L (A), FIS1 (B), MFF (C), MFN1 (D), MFN2 (E), and OPA1 (F) in WS5A, CP2A, and control astrocytes. Results are expressed as FPKM. (G) Representative images of western blotting for MFN1, OPA1, p-DRP1 (Ser 616), DRP1, MFF, and GAPDH, and quantitative measurements of protein levels of MFN1, OPA1, p-DRP1 (Ser 616), DRP1, and MFF in control and POLG-mutant astrocytes. (H) Representative images of live cell staining for MTG. The upper panel scale bar is $50 \mu \mathrm{m}$; the lower panel is taken from the upper panel and the scale bar is $10 \mu \mathrm{m}$. (I,J) Flow cytometric measurements of total MTDR level (I) and specific MTDR level normalized with MTG (J) in WS5A, CP2A, and control astrocytes. (K) Representative images of western blotting for TOMM20, PGC-1 $\beta$, GAPDH, and quantitative measurements of protein levels of TOMM20 and PGC-1 $\beta$ in control and POLG-mutant astrocytes. Data information: The data in A-F represent four different control astrocytes, including three clones from Detroit 551 control and one clone from AG05836 control, three clones from WS5A and two clones from CP2A. The data in G,K represent three different control astrocytes, including two clones from Detroit 551 control and one clone from AG05836 control, three clones from WS5A and three clones from CP2A. The data in $\mathbf{H}$ represent one clone from AG05836 control, one clone from WS5A and one clone from CP2A. The data in I,J represent three different control astrocytes, including two clones from Detroit 551 control and one clone from AG05836 control, three clones from WS5A and three clones from CP2A. Data are presented as mean \pm SEM for the number of samples ( $n \geq 3$ per clone). Mann-Whitney $U$ test was used for the data presented in A-K. Significance is denoted for $p$-values of less than 0.05. ${ }^{*} p<0.05 ;{ }^{* \star} p<0.01 ;{ }^{* \star *} p<0.001$; ${ }^{\star \star \star *} p<0.0001$; ns, not significant.
\end{abstract}

by Phyper ${ }^{3}$ based on hypergeometric test. Significant DEGs were defined as ones with at least 0.3 fragments per kilobase million (FPKM) level of expression in at least one of the conditions and a $q$-value less than 0.05 by Bonferroni test.

\section{Statistical Analyses}

All data are expressed as mean \pm SEM for the number of samples $(n \geq 3)$. Statistical analyses were performed with GraphPad Prism 8.0.2 software (GraphPad Software) using the Mann-Whitney $U$ test. Significance is denoted for $p$-values of less than 0.05 .

\section{RESULTS}

\section{Generation and Characterization of Patient-Specific Astrocytes Derived From Induced Pluripotent Stem Cells Carrying POLG Mutations}

To generate astrocytes, we generated iPSCs showing positive expression of SOX2, OCT4, and NANOG (Supplementary Figure 1) and then differentiated these into NSCs as described previously (Liang et al., 2020a,b, 2021). Two patient lines were used, one homozygous for c.2243G > C; p.W748S (WS5A) and the other a compound heterozygous for c.1399G > A/c.2243G > C; p.A467T/W748S (CP2A). We employed two control iPSC lines generated from normal human fibroblast lines, Detroit 551 and AG05836B for wildtype controls. In addition, one commercial human primary astrocyte line, HNA, was used as a positive astrocyte lineage control. We differentiated iPSC-derived NSCs to astrocytes as previously described (Liang et al., 2020a,b, 2021). The astrocytes from all patients and controls (Supplementary Figure 4) showed typical stellate morphology (Figure 1A). To minimize the intra-clonal phenotypic variability, multiple clones from each individual patient were differentiated and included in further studies.

Next, we characterized the iPSC-derived astrocytes using immunostaining against key astrocyte markers. We found robust expression of glial fibrillary acidic protein (GFAP) and S100 calcium-binding protein $\beta$ (S100 $\beta$ ) (Figure 1B and Supplementary Figure 2), and the functional markers excitatory

${ }^{3}$ https://en.wikipedia.org/wiki/Hypergeometric_distribution amino acid transporter 1 (EAAT-1, also known as GLAST-1) and glutamine synthetase (GS) (Figure 1C and Supplementary Figure 3). Astrocytic identity and purity were confirmed using flow cytometry against a panel of markers GFAP, S100ß, CD44, EAAT-1, and GS (Supplementary Figures 5-9). We found that over $99 \%$ of cells were positive for all these markers (Figure 1D). Additionally, we performed transcriptomic analysis to characterize the specific cell type and the level of astrocyte maturation from each individual clone. RNA sequencing (RNAseq) analysis was performed on three clones from WS5A, two clones from $\mathrm{CP} 2 \mathrm{~A}$, one clone from the commercial HNA control, and four control astrocyte clones including one clone from AG05836 and three clones from Detroit 551derived astrocytes.

Unsupervised hierarchical clustering showed that replicates from the individual clones in the control group clustered together, also clustering closely with HNA (Figure 1E). We found significant homogeneity with a cluster of astrocytic markers SOX9, NFIX, AQP4, ALDH1A1, GJA1, SLC1A3, $S 100 B$, and GFAP in all the samples, but no expression of neuronal markers NEROD1, NEUROG2, and RELN and oligodendrocyte markers OLIG1, OLIG2, SOX10, and NKX2.2 (Figure 1F).

Collectively, these data suggest that our protocol produces nearly pure cultures of differentiated astrocytes that exhibit similar transcriptional profiles to primary astrocytes.

\section{Transcriptomic Analysis Shows Downregulated Mitophagy/Autophagy Genes in POLG-Mutant Astrocytes}

We explored the DEGs as well as their related signaling and metabolic pathways by comparing the transcriptomic profile of patient (WS5A and CP2A) versus (vs.) control astrocytes. Using unsupervised clustering, we identified a clear clustering of the patient astrocytes (WS5A and CP2A) vs. controls (Figure 2A). The Venn diagram showed 547 genes were significantly differentially expressed in the WS5A lines and 509 in the CP2A lines compared to controls (statistical significance cutoff was $p=0.05$, Figure 2B).

KEGG metabolic pathway analysis revealed that the greatest enrichment was for genes involved in the mitophagy/autophagy pathways in both patient astrocyte lines (WS5A and CP2A) compared to controls (Figures 2C,D). This was 
also significant for genes involved in PI3K/AKT signaling pathway (Figures 2C,D). Unsupervised clustering of DEGs in the mitophagy/autophagy pathways demonstrated a similar pattern in WS5A cells vs. controls and CP2A cells vs. controls (Figure 2E). Differential analysis showed the downregulation of mitophagy genes in patient astrocytes compared to controls (Figure 2F). In addition, KEGG pathway enrichment analyses showed that there was also an associated dysregulation of both oxidative phosphorylation (Figure 2G) and other metabolic pathways, especially in NADH dehydrogenase-linked pathways (Figure 2H) in patient astrocytes. We also demonstrated significantly lower expressions of the mitophagy/autophagyrelated genes $M A P 1 L C 3 B$ (Figure 2I), SQSTM1 (Figure 2J), PINK1 (Figure 2K), and PRKN (Figure 2L) in both WS5A and $\mathrm{CP} 2 \mathrm{~A}$ lines compared to controls. This evidence suggests linkage between mitophagy changes and POLG-induced mitochondrial dysfunction in these astrocytes.

Given that mitochondrial dynamics also plays a role in mitochondrial quality control, we assessed transcript levels of mitochondrial fusion/fission-related genes. Expression levels of DNM1L, FIS1, MFF, MFN2, and OPA1 (Figures 3A-C,E,F) were similar in both patient-derived and control astrocytes, while expression of MFN1 was higher than control in WS5A patient astrocytes (Figure 3D). When we measured protein levels using western blotting, however, we found that levels of fusion proteins mitofusin-1 (MFN1) and optic atrophy (OPA1), as well as fission proteins mitochondrial fission factor (MFF) and dynamin-related protein 1 (DRP1), were unaffected in patient astrocytes (Figure 3G).

These results indicate that $P O L G$ mutations lead to changes in astrocytes that appear to target the mitophagy/autophagy pathways but not the mitochondrial fission/fusion pathways.

\section{POLG-Mutant Astrocytes Exhibit Loss of Mitochondrial Volume and Impaired Mitochondrial Function}

Next, we asked whether astrocytes generated from POLG patients exhibited changes in mitochondrial volume and function. We investigated mitochondrial morphology using fluorescence microscopy, and biogenesis with western blotting analysis, mitochondrial volume and mitochondrial membrane potential with flow cytometry. We assessed mitochondrial morphology using live cell staining with MTG and found a similar mitochondrial morphology in both patients and controls (Figure 3H).

We then investigated mitochondrial membrane potential. To do this, we used flow cytometry and the combination of the mitochondrial potential-dependent dye MTDR to measure membrane potential and a mitochondrial potentialindependent dye MTG to assess mitochondrial volume. We found significantly lower total MTDR (Figure 3I) and specific MTDR when normalized to mitochondrial mass MTG (Figure 3J) in both patient-derived astrocytes (WS5A and CP2A) as compared to controls.

Next, we quantified mitochondrial proteins by western blotting and observed decreased levels of TOMM20 in both patient lines (WS5A and CP2A) compared to controls (Figure 3K). To investigate if the decrease reflected downregulated mitochondrial biogenesis, we assessed PGC$1 \beta$, a positive regulator of mitochondrial biogenesis and respiration. We observed a significant decrease in PGC-1 $\beta$ in both patient-derived astrocytes (WS5A and CP2A) vs. controls (Figure 3K).

These findings demonstrate that POLG mutations in astrocytes are associated with loss of mitochondrial volume, decreased mitochondrial membrane potential, and biogenesis.

\section{POLG-Mutant Astrocytes Show Impaired Mitophagy Regulation via PINK1/Parkin Signaling Pathway}

Evidence from RNA-seq analysis suggested downregulation of mitophagy-related genes in patient iPSC-derived astrocytes. We investigated this further by treating astrocytes with carbonyl cyanide 4 - (trifluoromethoxy) phenylhydrazone (FCCP) - to induce mitophagy and then evaluated mitophagosome formation using a fluorescent mitophagy dye, together with a lysosome dye and the mitochondrial dye MTDR. We observed decreased red fluorescent puncta representing mitophagosome formation and green fluorescent intensity for measuring lysosomes in both patient and control astrocytes (Figure 4A). Quantification of the mean fluorescence intensity in more than five random areas showed that this decrease was significant for both mitophagy (Figure 4B) and lysosome formation (Figure 4B) in patient astrocytes. Consistent with the flow cytometric measurements (Figures 3I,J), MTDR staining demonstrated a significantly lower expression level in patient astrocytes as compared to controls (Figure 4B). These data suggest that astrocytes with $P O L G$ mutations lead to impaired mitophagy and decreased lysosome formation.

Next, we examined mitophagy-related proteins including the autophagosome marker microtubule-associated protein 1 light chain $3 \beta$ (LC3B), autophagy receptor p62, and lysosomal marker lysosomal-associated membrane protein 2A (LAMP2A) using western blotting. We found a decreased level of LC3B-II with a significantly decreased ratio of LC3B-II/LC3B-I in both WS5A and $\mathrm{CP} 2 \mathrm{~A}$ astrocytes compared to controls, indicating reduced mitophagy in POLG-mutant astrocytes (Figure 4C). We also identified the decreased protein expression of p62 (Figure 4C) and downregulation of LAMP2A (Figure 4C) in POLG-mutant astrocytes vs. control cells.

Loss mitochondrial membrane potential leads to activation of PINK1, which stimulates the recruitment of Parkin to the mitochondrial outer membrane of damaged mitochondria and activates Parkin's ubiquitin-ligase activity. Activated mitochondrial Parkin leads to the ubiquitination of mitochondrial proteins and subsequent mitophagy. We investigated PINK1/Parkin pathway using western blotting and found downregulation of PINK1 and Parkin in patient astrocytes as compared to controls (Figure $4 \mathrm{D}$ ), suggesting that $P O L G$ mutations may impair mitochondrial priming of mitophagy in astrocytes via PINK1/Parkin axis. 

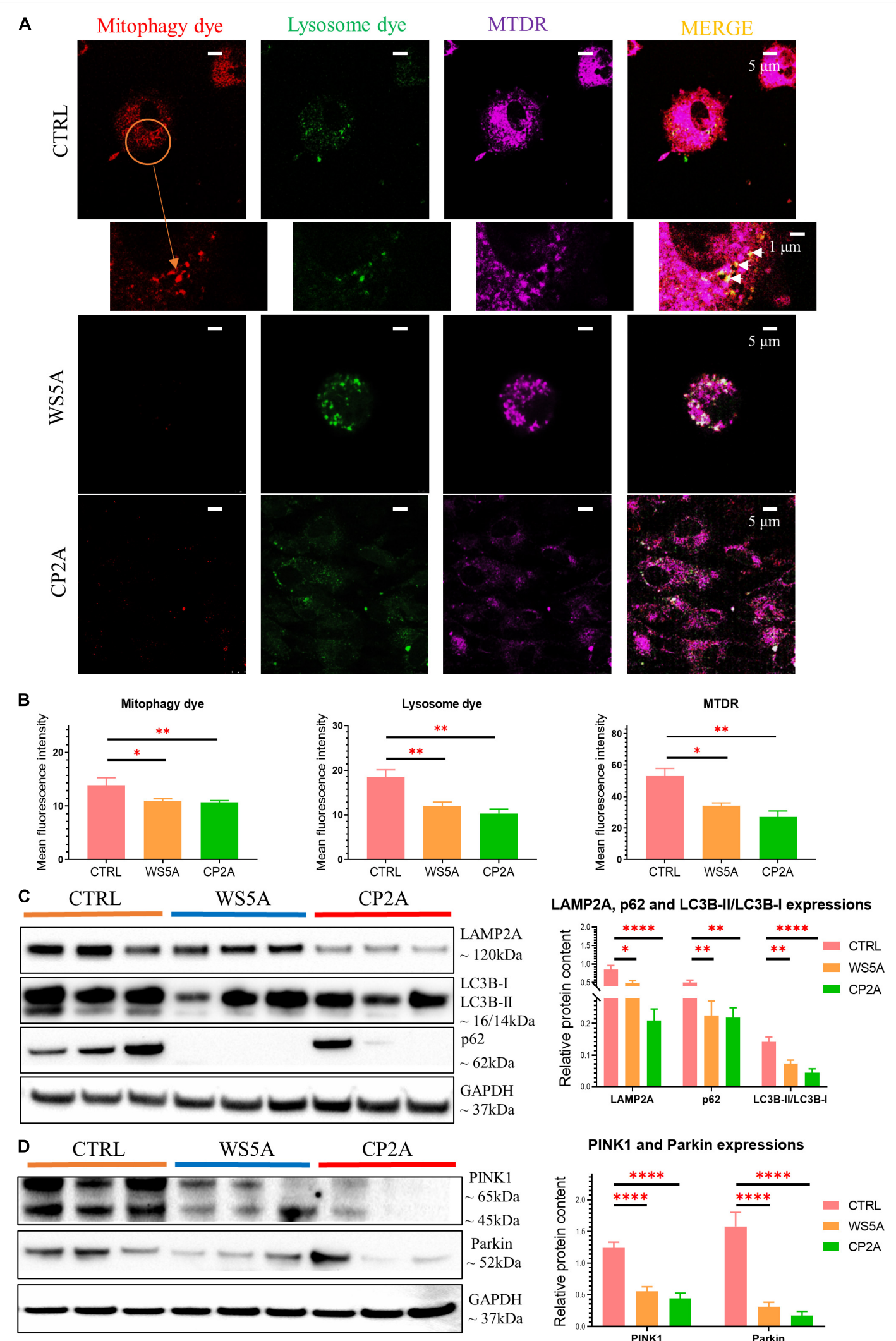

PINK1 and Parkin expressions

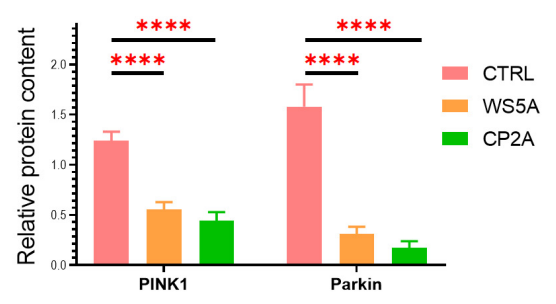

FIGURE $4 \mid$ (Continued) 
FIGURE 4 | POLG-mutant astrocytes show impaired mitophagy regulation via PINK1/Parkin signaling pathway. (A) Representative confocal images of immunostaining for mitophagy dye (red), lysosome dye (green), MTDR (purple), and MERGE (yellow) in WS5A, CP2A, and control astrocytes. Scale bar is 5 or $1 \mu \mathrm{m}$. White arrows denote mitophagy formation. The cells were treated with FCCP for $24 \mathrm{~h}$ before staining. (B) Quantification of the immunofluorescence levels of mitophagy dye, lysosome dye, and MTDR in WS5A, CP2A, and control astrocytes. (C) Representative images of western blotting for LAMP2A, p62, LC3B-I, LC3B-II, and quantitative measurements of protein levels in WS5A, CP2A, and control astrocytes. (D) Representative images of western blotting for PINK1, Parkin and quantitative measurements of protein levels of PINK1, Parkin in WS5A, CP2A, and control astrocytes. Data information: the data in A represent one clone from Detroit 551 control, one clone from WS5A, and one clone from CP2A. The data in B, 40,000 astrocytes per sample were seeded and four different control astrocytes, including three clones from Detroit 551 control and one clone from AG05836 control, two clones from WS5A, and two clones from CP2A were observed with three technical replicates. The data in C,D represent three different control astrocytes, including two clones from Detroit 551 control and one clone from AG05836 control, three clones from WS5A and three clones from CP2A. Data are presented as mean \pm SEM for the number of samples ( $n \geq 3$ per clone). Mann-Whitney $U$ test was used for the data presented in B-D. Significance is calculated by comparison to controls and is denoted for $p$-values of less than 0.05 . ${ }^{\star} p<0.05 ;{ }^{\star \star} p<0.01 ;{ }^{\star \star \star \star} p<0.0001$.

These findings suggest that POLG mutations can lead to impaired mitophagy and decreased lysosome formation via PINK1/Parkin-dependent pathway.

\section{POLG-Mutant Astrocytes Demonstrate Mitophagy Impairment via Modulating AKT/mTOR/AMPK/ULK1 Signaling Axis}

The mTOR signaling pathway plays a pivotal role in regulating metabolism (Lazarou et al., 2015; Perluigi et al., 2015). Abnormal activation of the AKT/mTOR/AMPK signaling pathway inhibits autophagy, and defects affecting this pathway are associated with human neurodegenerative disease (Saxton and Sabatini, 2017). Therefore, we asked if abnormal AKT/mTOR/AMPK signaling was driving the mitophagy impairment in POLG-mutant astrocytes. To address this question, we used western blotting to analyze p-mTOR, mTOR, p-AKT, AKT, p-ULK1, ULK1, p-AMPK (T183 + T172), and AMPK protein levels. We found a significantly increased ratio of $\mathrm{p}$-mTOR/mTOR in both patient astrocytes compared to controls, indicating abnormal activation of the mTOR signaling pathway (Figure 5A). We also demonstrated a significantly increased ratio of $\mathrm{p}$-AKT/AKT (Figure 5C) and a decrease in the ratio of $\mathrm{p}$-ULK1/ULK1 (Figure 5B) and p-AMPK $(\mathrm{T} 183+\mathrm{T} 172) /$ AMPK (Figure 5D).

Taken together, these data indicate that $P O L G$ mutations induced astrocytic mitophagy failure by regulating the AKT/mTOR/AMPK/ULK1 signaling pathway.

\section{Nicotinamide Riboside and Metformin Treatment Rescue Mitophagy Defect by Modulated SIRT1/AMPK/mTOR Pathway in POLG-Mutant Astrocytes}

Next, we asked whether the mitophagy defect could be rescued (Figure 6A). The $\mathrm{NAD}^{+}$precursor NR (Figure 6B) potentially activates mitophagy/autophagy through the SIRT1 or mTOR pathways (Cea et al., 2012; Fang et al., 2017; Vannini et al., 2019; Zheng et al., 2019). We therefore treated WS5A astrocytes for $72 \mathrm{~h}$ with varying concentrations of NR: $0,0.25,0.5,1$, and $2 \mathrm{mM}$. Cell viability was maintained under all treatment conditions (Supplementary Figure 10). Using western blotting, we found that cells treated with NR alone showed upregulated phosphorylation of SIRT1/SIRT1 (p-SIRT1/SIRT1) ratio at all drug concentrations. We also identified an increased p-AMPK (T183 + T172)/AMPK ratio, but only in cells treated with 0.25 and $0.5 \mathrm{mM} \mathrm{NR}$ (Figure 6D). No significant change in the mTOR pathway (Figure 6D) or the mitophagy-related proteins LC3B-II/LC3B-I and p62 (Figure 6E) were identified.

The AMPK activator metformin (Figure 6C) improves mitophagy (Bhansali et al., 2020; Bharath et al., 2020; Kodali et al., 2021) and mitochondrial function (Bharath et al., 2020) via AMPK pathway activation. Therefore, we treated WS5A astrocytes for $72 \mathrm{~h}$ with a series of metformin concentrations: $0,1.25,2.5,5$, and $10 \mathrm{mM}$. We observed that cell viability was maintained under all treatment conditions (Supplementary Figure 11). We found upregulated AMPK signaling with increased phosphorylation of AMPK at T183 + T172/AMPK ratio at all concentrations (Figure 6F). Metformin-treated cells showed a dose-dependent decrease in phosphorylated mTOR relative to total mTOR (Figure 6F) and an increased LC3B-II/LC3B-I ratio, which peaked in cells treated with $2.5 \mathrm{mM}$, and increased $\mathrm{p} 62$ at all concentrations except $10 \mathrm{mM}$ (Figure 6G).

We then examined whether combining NR and metformin gave additional benefit. Cells were treated with the following concentrations: $0 \mathrm{mM}$, NR $0.25 \mathrm{mM}+$ metformin $1.25 \mathrm{mM}$, NR $0.5 \mathrm{mM}+$ metformin $2.5 \mathrm{mM}$, NR $1 \mathrm{mM}+$ metformin $5 \mathrm{mM}$, and NR $2 \mathrm{mM}+$ metformin $10 \mathrm{mM}$. Cell growth was maintained under all treatment conditions (Supplementary Figure 12). Combined treatment gave increased levels of p-SIRT1/SIRT1 ratio and p-AMPK (T183 + T172)/AMPK ratio, but decreased level of p-mTOR/mTOR ratio at all concentrations (Figure 7A). Dual treatment increased LC3B-II/LC3B-I ratio, showing a peak in the cells treated with $0.5 \mathrm{mM} \mathrm{NR}$ and $2.5 \mathrm{mM}$ metformin, while $10 \mathrm{mM}$ did not reach significance (Figure 7B). The level of p62 increased at all concentrations (Figure 7B).

To confirm whether the dual treatment could rescue the mitophagy, we treated both WS5A and CP2A astrocytes and performed the mitophagy detection assay described above. When incubated with FCCP for $24 \mathrm{~h}$, we observed increased mitophagy (higher red fluorescent signal) and lysosomal formation (elevated green, fluorescent puncta) in the cotreated patient cells as compared to controls (Figure 7C). Quantification of the fluorescent signals showed significantly increased levels for both mitophagy and lysosomal formation (Figures 7D,E) in both patient iPSC-derived astrocytes after the treatment. 


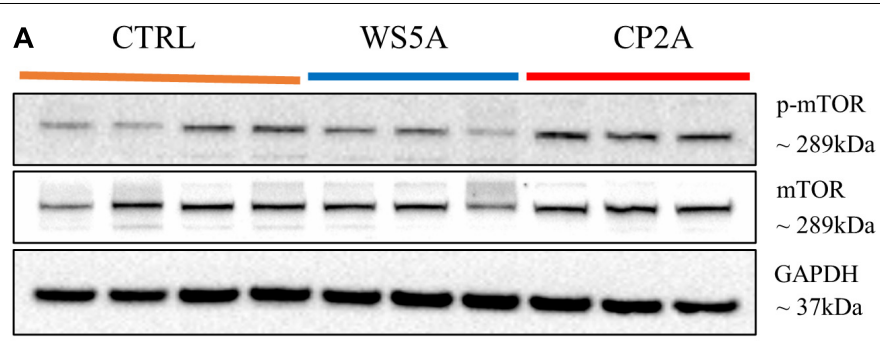

p-mTOR, mTOR and p-mTOR/mTOR expressions

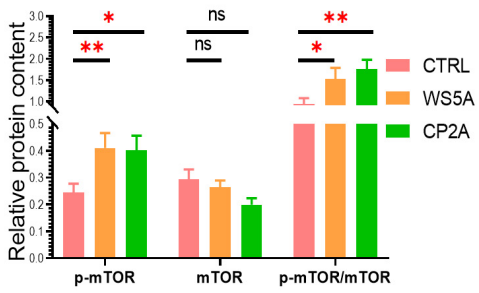

B

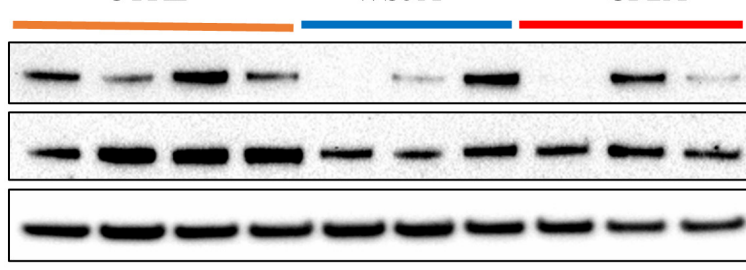

$\mathrm{p}$-ULK1

$\sim 140 \mathrm{kDa}$

ULK1

$\sim 150 \mathrm{kDa}$

GAPDH

$\sim 37 \mathrm{kDa}$
p-ULK1, ULK1 and p-ULK1/ULK1 expressions

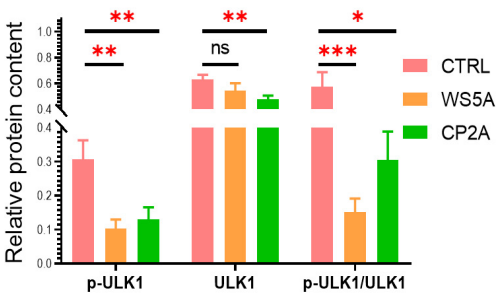

p-AKT, AKT and p-AKT/AKT expressions

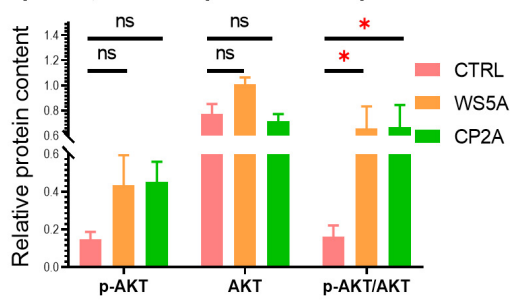

D

CTRL

WS5A

CP2A

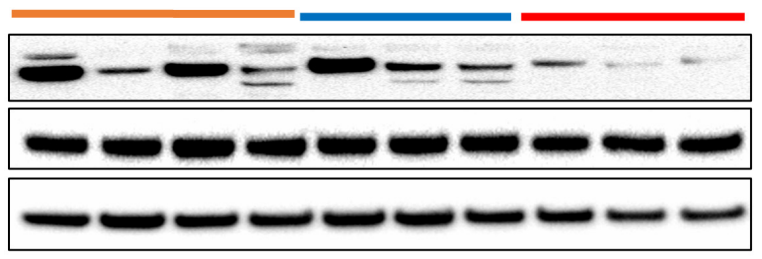

p-AMPK

$(\mathrm{T} 183+\mathrm{T} 172)$

$\sim 64 \mathrm{kDa}$

AMPK

$\sim 62 \mathrm{kDa}$

GAPDH

$\sim 37 \mathrm{kDa}$

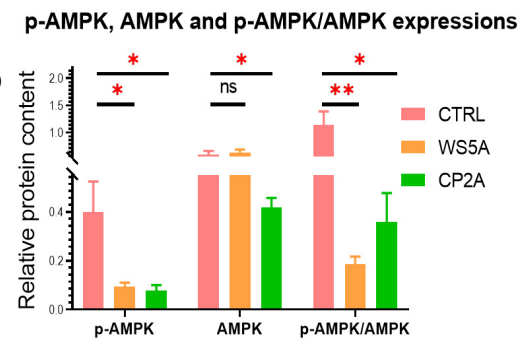

FIGURE 5 | POLG-mutant astrocytes demonstrate mitophagy impairment via modulating AKT/mTOR/AMPK/ULK1 signaling axis. (A) Representative images of western blotting for p-mTOR, mTOR, GAPDH, and quantitative measurements of protein levels in WS5A, CP2A, and control astrocytes. (B) Representative images of western blotting for p-ULK1, ULK1, GAPDH, and quantitative measurements of protein levels in WS5A, CP2A, and control astrocytes. (C) Representative images of western blotting for p-AKT, AKT, GAPDH, and quantitative measurements of protein levels of in WS5A, CP2A, and control astrocytes. (D) Representative images of western blotting for p-AMPK (T183 + T172), AMPK, GAPDH, and quantitative measurements of protein levels in WS5A, CP2A, and control astrocytes. Data information: the loading controls from $\mathbf{D}$ are re-used from B,C. The data in $\mathbf{A}-\mathbf{D}$ represent four different control astrocytes, including three clones from Detroit 551 control and one clone from AG05836 control, three clones from WS5A and three clones from CP2A. Data are presented as mean \pm SEM for the number of samples ( $n \geq 3$ per clone). Mann-Whitney $U$ test was used for the data presented in $\mathbf{A}-\mathbf{D}$. Significance is denoted for $p$-values of less than $0.05 .{ }^{*} p<0.05 ;{ }^{* *} p<0.01$; ${ }^{* \star *} p<0.001 ;$ ns, not significant.

Collectively, our data suggest NR alone and metformin alone modulate mitophagy in POLG-mutant astrocytes in different manners, while dual treatment of NR and metformin provides a quantifiable improvement of the mitophagy impairment via regulating SIRT1/AMPK/mTOR signaling pathway in POLGmutant astrocytes. 
A
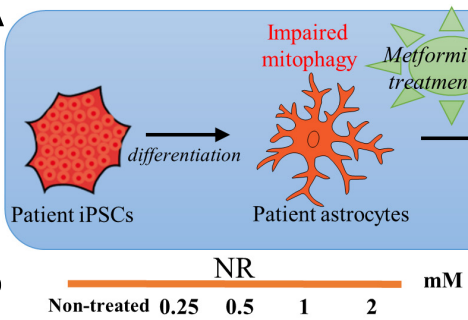

$-0-0.20$ $-\mathbf{m}={ }_{\sim 120 \mathrm{kDa}}^{\mathrm{p}-\mathrm{STTT}}$ $-\boldsymbol{m - m}{ }_{\sim 120 \mathrm{kDa}}^{\mathrm{SIRT}}$ $\mathbf{m a t}=\underset{\sim 64 \mathrm{kDa}}{\mathrm{p}-\mathrm{AMPK}}$ AMPK p-mTOR $\Longrightarrow-1=\sim_{2289 \mathrm{kD}}^{\mathrm{p}-\mathrm{mTOR}}$ $-2-1 \mathrm{mTOR}$ GAPDH

E

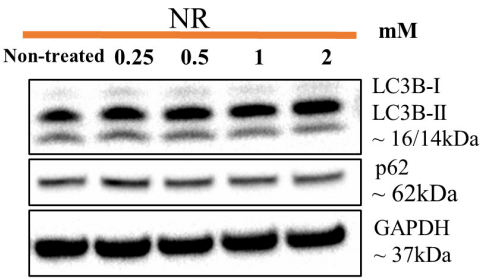

$\mathbf{F}$

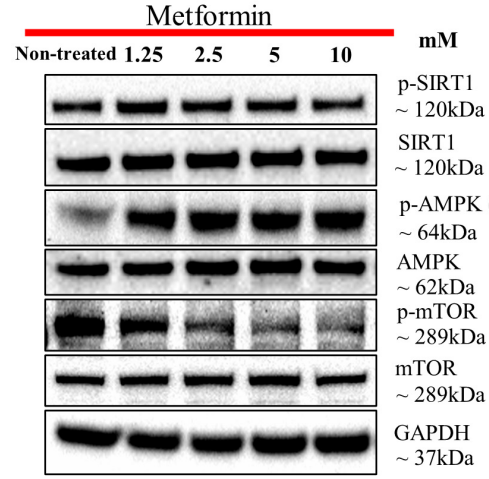

G

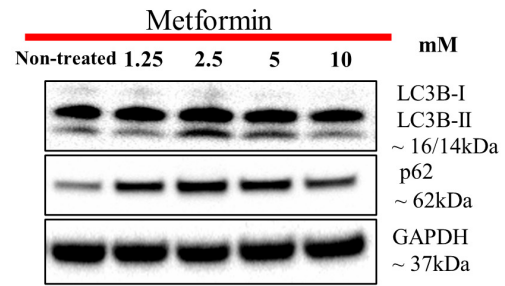

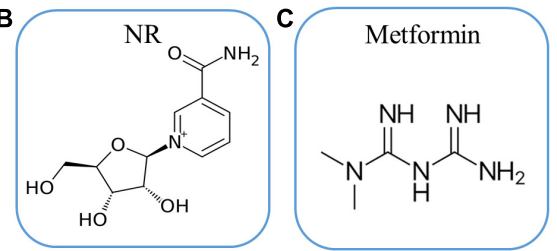

Protein expressions with NR treatment

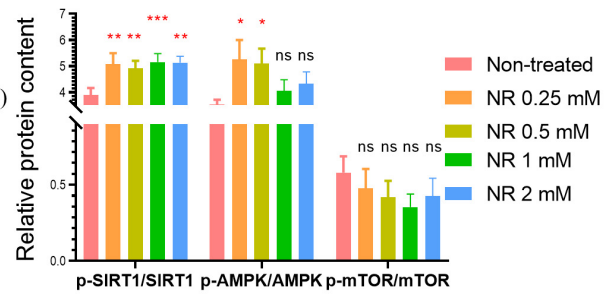

Protein expressions with NR treatment

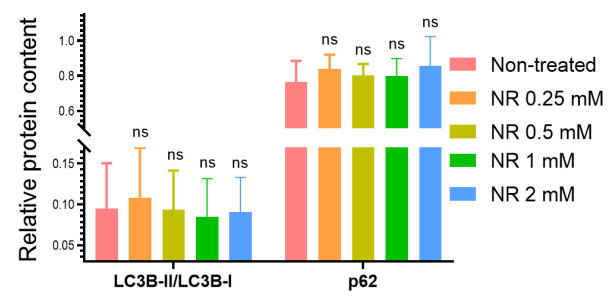

Protein expressions with metformin treatment

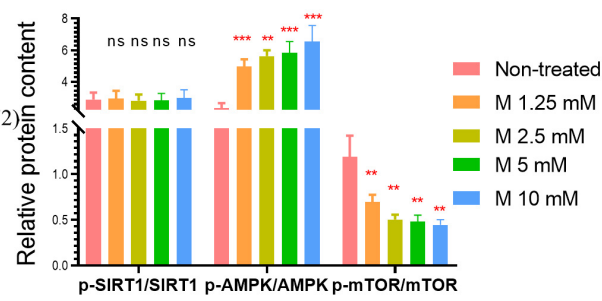

Protein expressions with metformin treatment

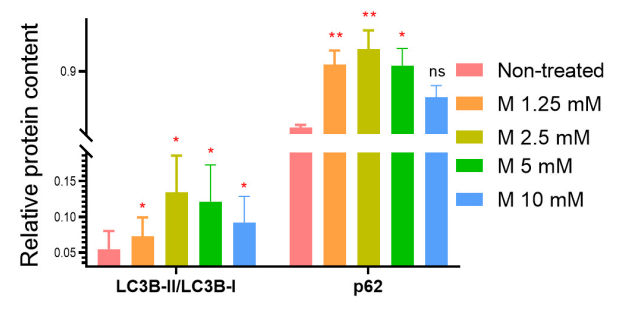

FIGURE 6 | Nicotinamide riboside and metformin treatment modulated SIRT1/AMPK/mTOR pathway in POLG-mutant astrocytes. (A) Schematic diagram of compound treatment for POLG-mutant astrocytes. (B,C) Chemical structure of NR (B) and metformin (C). (D) Representative images and quantification of western blotting for p-SIRT1, SIRT-1, p-AMPK (T183 + T172), AMPK, p-mTOR, mTOR, and GAPDH in the POLG WS5A astrocytes treated with a serial concentration of NR alone for $72 \mathrm{~h}$. (E) Representative images and quantification of the western blotting for LC3B-I, LC3B-II, p62, and GAPDH in the POLG WS5A astrocytes treated with a serial concentration of NR alone for $72 \mathrm{~h}$. (F) Representative images and quantification of western blotting for p-SIRT1, SIRT1, p-AMPK (T183 + T172), AMPK, p-mTOR, mTOR, and GAPDH in the POLG WS5A astrocytes treated with a serial concentration of metformin alone for $72 \mathrm{~h}$. (G) Representative images and quantification of the western blotting for LC3B-I, LC3B-II, p62, and GAPDH in the POLG WS5A astrocytes treated with a serial concentration of metformin alone for $72 \mathrm{~h}$. Data information: the data in $\mathbf{D}-\mathbf{G}$ represent one clone from WS5A. Mann-Whitney $U$ test was used for the data presented in $\mathbf{D}-\mathbf{G}$. Significance is calculated by comparison to non-treated cells and is denoted for $p$-values of less than $0.05 .{ }^{*} p<0.05$; ${ }^{* *} p<0.01$; ${ }^{* \star *} p<0.001$; ns, not significant. 


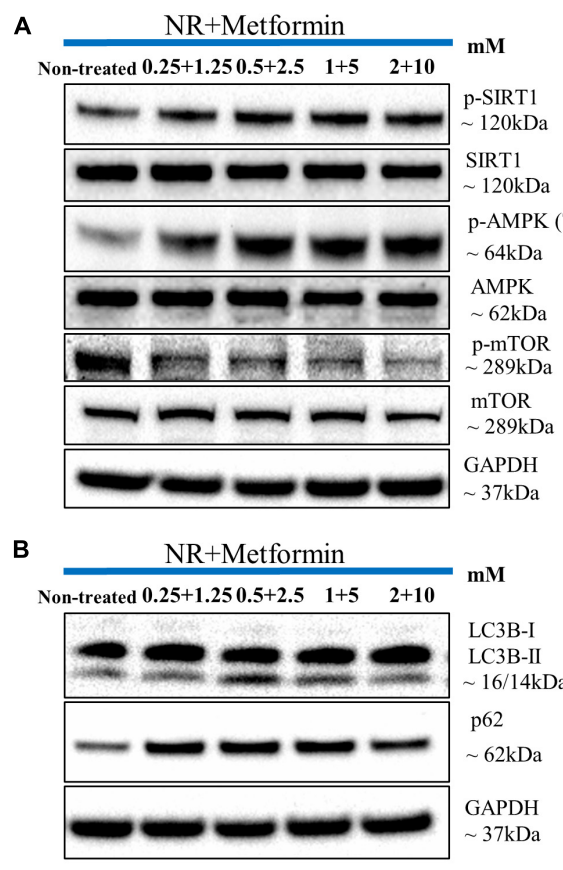

C
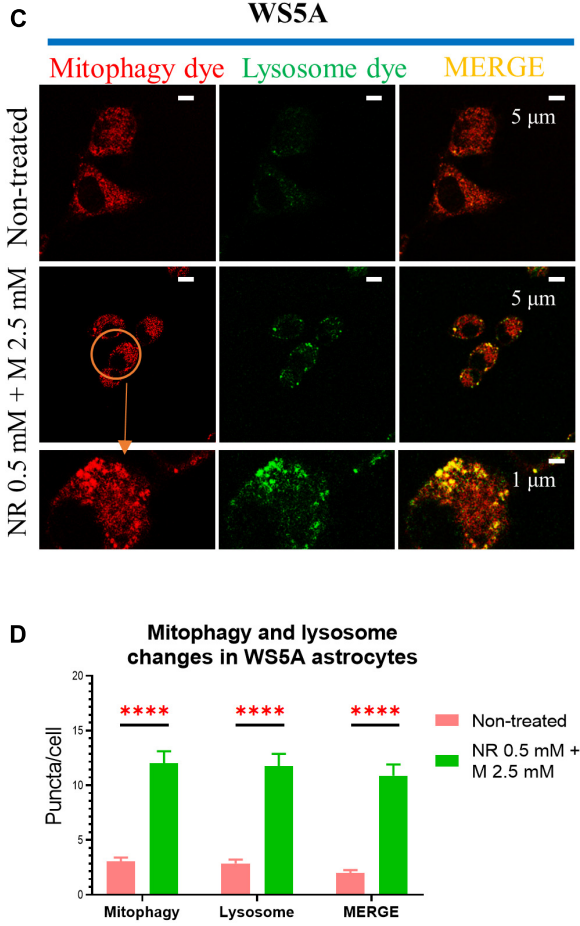
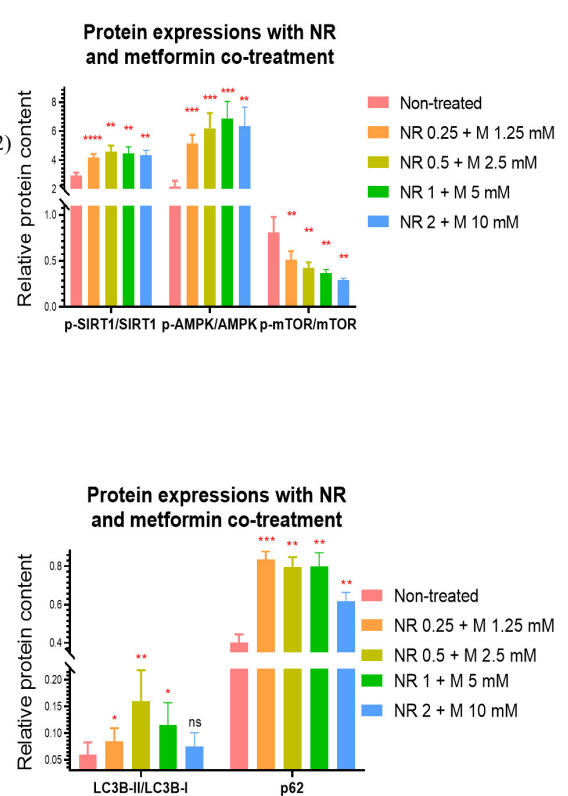

CP2A
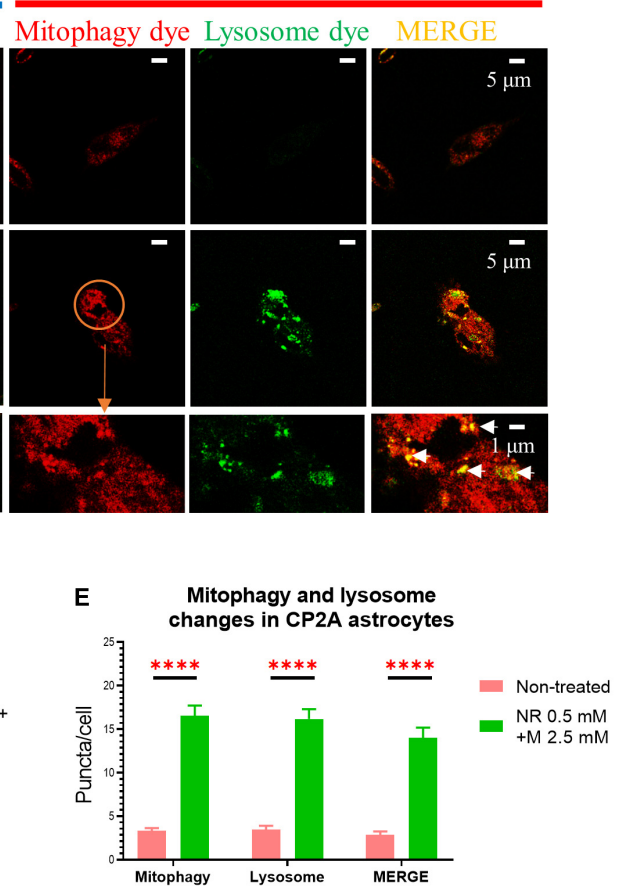

FIGURE 7 | Nicotinamide riboside and metformin co-treatment rescue mitophagy defect in POLG-mutant astrocytes. (A) Representative images and quantification of western blotting for p-SIRT1, SIRT1, p-AMPK (T183 + T172), AMPK, p-mTOR, mTOR, GAPDH, and in the POLG WS5A astrocytes treated with a serial concentration of NR and metformin co-treatment for 72 h. (B) Representative images and quantification of the western blotting for LC3B-I, LC3B-II, p62, and GAPDH in the POLG WS5A astrocytes treated with a serial concentration of NR and metformin co-treatment for $72 \mathrm{~h}$. (C) Representative confocal images of immunostaining for mitophagy dye (red), lysosome dye (green), and MERGE (yellow) in WS5A and CP2A astrocytes with and without NR and metformin dual treatment. Scale bar is 5 or $1 \mu \mathrm{m}$. White arrows denote mitophagy formation. (D,E) Quantification of the puncta numbers/cell of mitophagy dye, lysosome dye, and MERGE in WS5A (D) and CP2A astrocytes (E) with and without NR and metformin co-treatment. Data information: the data in A,B represent one clone from WS5A. The data in $\mathbf{C}$ represent one clone from WS5A with and without co-treatment, one clone from CP2A with and without co-treatment. The data in D,E, 40,000 astrocytes per sample were seeded and two clones from WS5A with and without co-treatment and two clones from CP2A with and without co-treatment were observed with three technical replicates. Mann-Whitney $U$ test was used for the data presented in $\mathbf{A}, \mathbf{B}, \mathbf{D}, \mathbf{E}$. Significance is calculated by comparison to non-treated cells and is denoted for $p$-values of less than 0.05. ${ }^{\star} p<0.05 ;{ }^{\star \star} p<0.01 ;{ }^{\star \star \star} p<0.001 ;{ }^{\star \star \star \star} p<0.0001$; ns, not significant. 


\section{Nicotinamide Riboside and Metformin Treatment Improve Mitochondrial Function in POLG-Mutant Astrocytes}

Metformin is a known inhibitor of respiratory chain complex I, but this has been shown to be concentration dependent (Owen et al., 2000). There is convincing evidence that inhibition of complex I occurs only at higher metformin concentrations, while at lower concentrations, it improves mitochondrial respiratory chain function (Wang et al., 2019). To check the effect of our treatment, we treated cells with NR, metformin, or both using the following concentrations: $0 \mathrm{mM}$, NR $0.25 \mathrm{mM}+$ metformin $1.25 \mathrm{mM}$, NR $0.5 \mathrm{mM}+$ metformin $2.5 \mathrm{mM}$, NR $1 \mathrm{mM}+$ metformin $5 \mathrm{mM}$, and NR $2 \mathrm{mM}+$ metformin $10 \mathrm{mM}$ and measured the complex I subunit NDUFB10 expression using western blotting. We observed increased complex I expression with metformin alone or co-treatment when this drug was used at lower concentrations (Figures 8A,C,D). In addition, we found increased complex I enzyme activity in cells treated with metformin $1.25 \mathrm{mM}$, but not with other concentrations (Supplementary Figure 13). To investigate whether NR and metformin treatment could improve the mitochondrial biogenesis in POLG-mutant astrocytes, we then examined PGC-1 $\alpha$ expression using western blotting and found that PGC- $1 \alpha$ also tended to increase in patient astrocytes under all treatment conditions, but only at the concentration of the NR $0.5 \mathrm{mM}+$ metformin $2.5 \mathrm{mM}$ and NR $1 \mathrm{mM}+$ metformin $5 \mathrm{mM}$ with significant statistical significance (Figures 8A-D).

Next, we checked the effect of treatment on cell growth, mitochondrial mass, and membrane potential. We treated astrocytes with $0.5 \mathrm{mM} \mathrm{NR}$ and $2.5 \mathrm{mM}$ metformin, the concentrations demonstrating the most significant effect as defined in earlier assays. We found that combined treatment did lead to an increase in cell number (Figures 8E,F), mitochondrial mass (Figure 8G), and mitochondrial membrane potential; both total (Figure $\mathbf{8 H}$ ) and specific levels normalized to mass (Figure 8I) although the increase was small.

Taken together, these data indicate that NR and metformin treatment improved the mitochondrial biogenesis and functions in POLG-mutant astrocytes, suggesting the mitophagy enhancers as potential treatments for POLG-related disorders.

\section{DISCUSSION}

POLG mutations impair the replication of mtDNA and cause a range of mitochondrial diseases many of which affect the brain (Hikmat et al., 2020). We showed it was possible to replicate the cellular phenotypes of mitochondrial dysfunction and abnormal $\mathrm{NAD}^{+}$metabolism in neural cells (Liang et al., 2020b, 2021) and confirmed these changes in iPSC-derived astrocytes (Liang et al., 2020a). Guided by unsupervised transcriptomic analysis, which indicated a major dysregulation of mitophagy/autophagy, we found that $P O L G$ mutations were clearly associated with impaired mitophagy in astrocytes. This impairment occurs via multiple mechanisms including (a) downregulation of the autophagic/mitophagic protein p62 and reduced lipidation of LC3B-II, (b) impaired autophagosome-lysosome fusion, (c) downregulation of the PINK1/Parkin pathway, and (d) dysregulation of AKT/mTOR/AMPK/ULK1 pathway. Further and most notably, we demonstrate that NR and metformin treatment improved the impaired mitophagy machinery and appeared to restore the mitochondrial defect. This opens new possibilities for the treatment of these disorders by using mitophagy-enhancing reagents.

In our study, we chose healthy, age-matched control iPSCs and patient-specific iPSCs then differentiated them into astrocytes using a modified technique (Liang et al., 2020a,b, 2021). We are fully aware that the current state of the art for iPSC studies is to use gene-corrected isogenic controls; however, the presence of compound mutations, such as those used in this study (CP2A mutation), makes the generation of individual controls impracticable. At the same time, it has been stated that the high efficiency of genome cutting and repair makes the introduction of heterozygous alleles by the standard CRISPR/Cas9 technique near impossible (EauClaire and Webb, 2019; Liang et al., 2020a,b, 2021).

We performed RNA-seq analysis to confirm the cellular identity and screen for changes in cellular pathways affected by the presence of POLG mutations in astrocytes (Liang et al., 2020a). We found that both patient and control astrocytes showed similar astrocytic lineage profiles. Pathway analysis showed clear dysregulation of genes involved in mitophagy/autophagy, with both patient lines being downregulated. This was confirmed by assessing the individual genes involved in mitophagy/autophagy. We identified multiple downregulated genes in several mitophagy/autophagy-related pathways including the PI3K/AKT signaling pathway and cAMP signaling pathway as well as metabolic pathways. While mitochondrial fusion and fission are important activities for the removal of defective organelles via mitophagy, we observed similar transcriptomic profiles in patient and control astrocytes. Our data therefore suggest that while POLG mutations lead to the downregulation of mitophagy/autophagy-related genes, this appears to be independent of mitochondrial fission/fusion.

To investigate how dysregulated mitophagy might impact POLG affected mitochondria, we looked at changes in mitochondrial function including mitochondrial volume, membrane potential, and biogenesis. We found that POLGmutant astrocytes had reduced mitochondrial volume, lower membrane potential, and decreased mitochondrial biogenesis factor PGC-1 $\beta$. This suggests that POLG not only disrupts mitophagy regulation but might also impair mitochondrial biogenesis. Thus, our study suggests that mitochondrial biogenesis and mitophagy are coordinated to regulate mitochondrial homeostasis and maintain cellular energy levels in tissues such as the brain.

Consistent with the transcriptomic findings, we observed that mitophagy activity was diminished under FCCP-induced conditions in both patient cell lines. When we measured the LC3 conversion (LC3B-I-LC3B-II) by western blot analysis, the steady-state levels of LC3B-II were significantly lower in both patient lines. There was also a lower expression of p62. Autophagic adapter protein p62 is the substrate of Parkin 

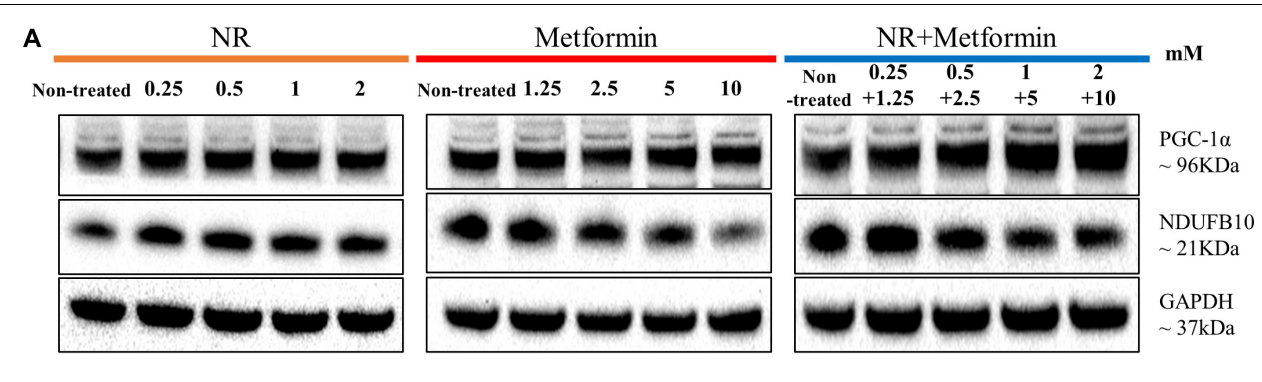

B

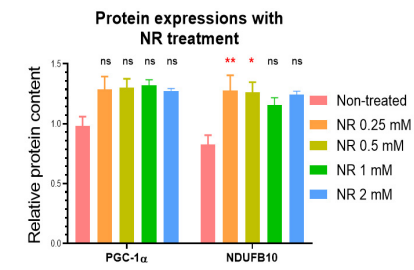

C Protein expressions with metformin treatment

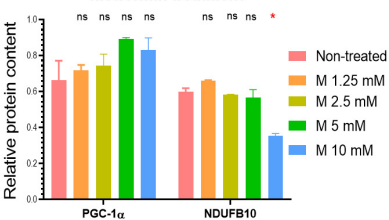

D Protein expressions with NR

and metformin co-treatment

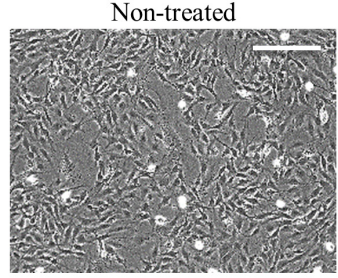

NR $0.5 \mathrm{mM}+$ Metformin $2.5 \mathrm{mM}$

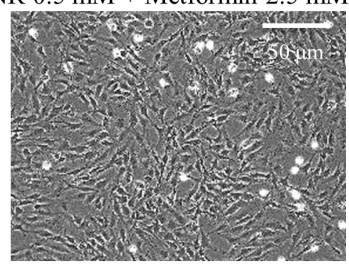

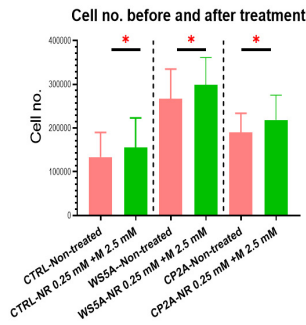

H

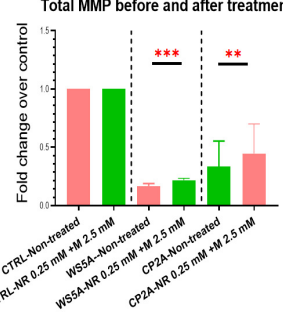

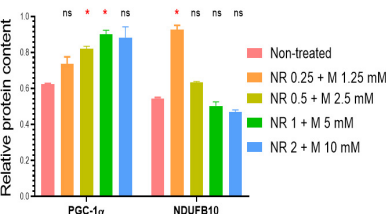

G MTG before and after treatment

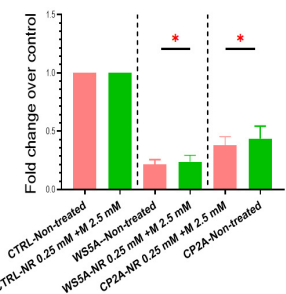

I Specific MMP before and after treatment

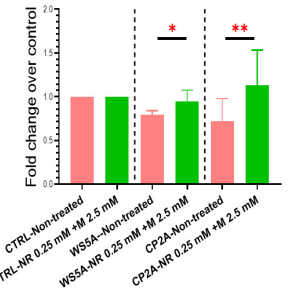

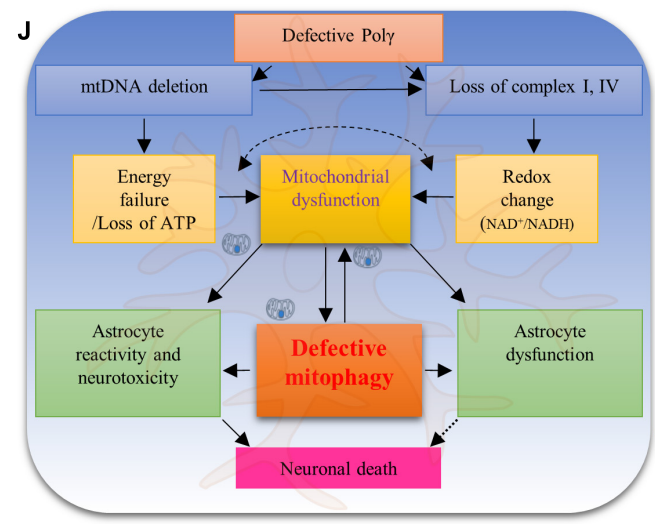

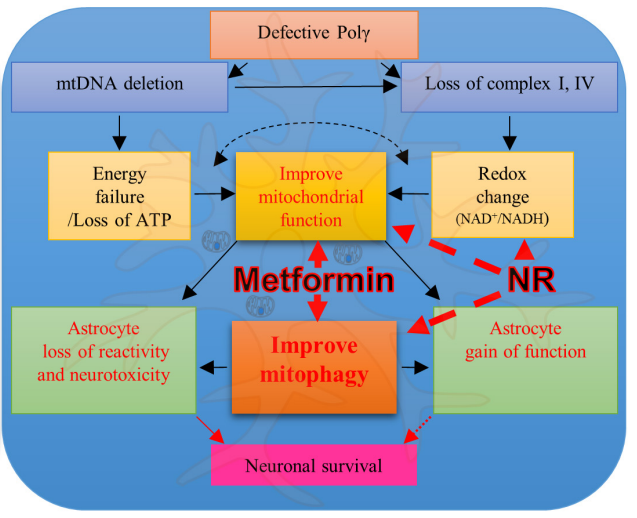

FIGURE 8 | Nicotinamide riboside and metformin treatment improve mitochondrial function in POLG-mutant astrocytes. (A) Representative images of the western blotting for NDUFB10, PGC-1 $\alpha$, and GAPDH in the POLG WS5A astrocytes treated with a serial concentration of NR alone, metformin alone, and co-treatment for 72 h. (B-D) Protein quantifications of the western blotting in A for NDUFB10 and PGC-1 $\alpha$ in the POLG WS5A astrocytes treated with a serial concentration of NR alone (B), metformin alone (C), and co-treatment (D) for $72 \mathrm{~h}$. (E) Representative phase-contrast images of WS5A astrocytes before and after the dual treatment of NR $0.5 \mathrm{mM}$ and metformin $2.5 \mathrm{mM}$. Scale bar is $50 \mu \mathrm{m}$. (F) Cell number counting of WS5A astrocytes before and after the dual treatment of NR $0.5 \mathrm{mM}$ and metformin 2.5 mM. (G-I) Flow cytometric analysis for MTG (G) and MMP at total level measured by TMRE (H) and specific MMP level calculated by total TMRE/MTG (I) of WS5A astrocytes before and after the dual treatment of NR 0.5 mM and metformin $2.5 \mathrm{mM}$. MTG, MMP, and specific MMP were normalized with non-treated cells. (J) Summary of the possible disease mechanisms in astrocyte defects and neuronal loss in POLG-related disorders. Data information: the data in A-I represent one clone from WS5A. Mann-Whitney $U$ test was used for the data presented in B-D and $\mathbf{F}-\mathbf{I}$. Significance is calculated by comparison to non-treated cells and is denoted for $p$-values of less than 0.05. ${ }^{*} p<0.05 ;{ }^{* *} p<0.01 ;{ }^{* \star *} p<0.001$; ns, not significant. 
and is required for Parkin-induced mitophagy (Song et al., 2016). Thus, aberrant p62 could influence the balance of mitophagy and further disturb mitochondrial quality control. Accumulation of misfolded proteins is known to induce aberrant p62 expression, and p62 is involved in multiple pathways associated with neurodegeneration (Homma et al., 2014; Ma et al., 2019). Abnormal expression of p62 can induce mitophagy and autophagy dysfunction, which can further accelerate misfolded protein aggregation. Based on our study, the data suggest that p62 is associated with POLG-related diseases and impaired Parkin-mediated mitophagy. To dissect what was driving the impairment of mitophagy, we examined the molecular signaling pathways involved. We showed that POLG mutations impaired mitochondrial priming of mitophagy via the PINK1/Parkin pathway. In mammalian cells, lower mitochondrial membrane potential leads to accumulation of PINK1 on the outer mitochondrial membrane (OMM) and subsequent recruitment of the E3 ubiquitin ligase Parkin. Parkin-mediated ubiquitination of OMM proteins serves as a general signal recognized by autophagy adaptor proteins, such as p62, which further target LC3B and ubiquitin chains, translocating these dysfunctional mitochondria into phagophores. We previously demonstrated the downregulation of the PINK1/Parkin pathway and showed that POLG mutations drive a lower level of ATP in astrocytes (Liang et al., 2020a). Given that ATP is a key regulator of PINK1-mediated mitophagy by controlling PINK1 translation levels (Lee et al., 2015), we suggest that POLG-induced ATP depletion downregulates the translation of PINK1 and therefore the recruitment of Parkin in response to mitochondrial depolarization.

We also detected reduced lysosome formation in POLGmutant astrocytes following FCCP treatment and a reduction in the protein level of lysosome-related membrane protein LAMP2A, indicating damage or dysfunction of lysosomes. Damaged mitochondria are degraded by the autophagylysosome pathway (Plotegher and Duchen, 2017), and studies in PARK2-PD fibroblasts suggest that lysosomal function may be influenced by mitochondrial quality control (Guerra et al., 2019). Our data suggest that mutations in POLG induce mitochondrial dysfunction that leads to lysosomal impairment and impairment of mitophagosome-lysosome fusion. Lysosomal impairment, in turn, can trigger mitophagy failure and functional mitochondrial defects in astrocytes. In addition, LAMP2A mediates lysosome fusion and is essential for the final stage of mitophagy. A recent study showed the LAMP2A is required for the clearance of mitochondria but is dispensable for PINK1/Parkin-mediated mitophagy (Liu et al., 2020). We thus conclude that $P O L G$ mutations may impair the mitophagy machinery in astrocytes in both a PINK1/Parkin-dependent and independent manner, which includes impairment of autophagylysosome pathway.

Our previous studies showed that POLG-mutant astrocytes generated less ATP and significantly higher levels of lactate than controls indicating a shift from OXPHOS to glycolysis (Liang et al., 2020a). Given that AMPK is a key metabolic sensor/regulator and essential to balance glycolysis and mitochondrial metabolism in order to control cellular stress and survival (Kishton et al., 2016), we speculated that this metabolic shift could inhibit the AMPK signaling pathway. This, in turn, would modulate mTOR signaling via its upstream activator AKT and the downstream ULK1 signaling leading to downregulation of the mitophagy machinery. To investigate how the AMPK, mTOR, and PI3K/AKT pathway might impact POLG-affected mitophagy, we investigated changes in phosphorylated AMPK and mTOR, as well as the upstream PI3K/AKT proteins. We found, as expected, that POLG-mutant astrocytes showed suppressed AMPK and activated mTOR via upstream PI3K/AKT proteins. Similar to our findings, studies have demonstrated that autophagy in PD dopaminergic cells was regulated by the AMPK/mTOR signaling pathway, suggesting that AMPK/mTOR-mediated autophagy may also be a potential therapeutic target for the treatment of PD patients (Ng et al., 2012). Together, our findings support the suggestion that changes in the AMPK/mTOR signaling pathway contribute to the POLG-induced mitophagy dysfunction.

Previously, we reported that POLG-mutant astrocytes exhibited abnormal $\mathrm{NAD}^{+}$metabolism via SIRT1-dependent regulation (Liang et al., 2020a). SIRT1 is a highly conserved $\mathrm{NAD}^{+}$-dependent protein deacetylase that has emerged as a critical mitophagy regulator (Fang et al., 2014). SIRT1 is also required for AMPK activation (Price et al., 2012) and can induce mitophagy activity via regulating AMPK/mTOR/ULK signaling (Mei et al., 2020). Recent work has suggested that supplementation with the $\mathrm{NAD}^{+}$precursor $\mathrm{NR}$ can potentially restore $\mathrm{NAD}^{+}$metabolic profiles and improve mitophagy in a ULK1-dependent manner (Fang et al., 2019a). We therefore treated our patient astrocytes with NR to increase SIRT1 activity and investigated the effects on AMPK/mTOR-dependent mitophagy. Despite finding that SIRT1 phosphorylation was increased, NR treatment alone failed to alter AMPK/mTOR-related proteins or rectify the mitophagy defects in POLG-mutant astrocytes.

Studies have also suggested that metformin might be beneficial by maintaining SIRT1 activity during the aging process when $\mathrm{NAD}^{+}$levels decline (Bhansali et al., 2020). Moreover, previous studies showed that metformin upregulated mitophagy markers and enhanced mitophagic flux through the AMPK pathway (Bhansali et al., 2020). Our studies showed that metformin activated AMPK and mTOR, but did not increase SIRT1 activity. Metformin stimulated mitophagy in POLG-mutant astrocytes as evidenced by increased LC3B conversion and p62 expression. Thus, given that metformin and NR appeared to activate different parts of the mitophagy process, we asked whether dual treatment would ameliorate the mitophagy defects in POLGmutant astrocytes. Our results suggest that this is the case: not only does dual treatment significantly elevate LC3B-II/LC3B-I ratio and $\mathrm{p} 62$ level, but it also improves mitophagy and lysosome formation in POLG-mutant astrocytes.

Metformin is known to inhibit complex I, but this is concentration dependent: at higher concentrations it inhibits, while at lower concentrations it appears to improve mitochondrial respiratory chain function (Wang et al., 2019). In our study, we showed an increased complex I expression with NR 
and lower concentration metformin co-treatment. Mitochondrial biogenesis and mitochondrial functional properties were also increased in patient astrocytes following exposure to the cotreatment. In an earlier study (Liang et al., 2020b), we showed that POLG mutations lead to mtDNA depletion and loss of complexes I and IV in astrocytes. These changes also led to ATP depletion and abnormal $\mathrm{NAD}^{+}$metabolism. Our current studies demonstrate that POLG-mutant astrocytes also have impaired mitophagy (Figure 8J). It is possible to hypothesize, therefore, that the neuronal loss associated with POLG mutations might occur via two mechanisms; first, loss of normal mitochondrial function and disrupted mitophagy impairs normal astrocytic support function, and second, the mitochondrial defects cause astrocytes to become reactive and neurotoxic. Our work suggests that treatment of NR and metformin potentially improves both the mitochondrial functional deficit and mitophagy offering a way of rescuing the disease phenotype in patients with POLG mutations.

\section{CONCLUSION}

Overall, these results identify that $P O L G$ mutations lead to accumulation of damaged mitochondria which can further impart the mitophagy machinery by regulating the PINK1/Parkin and $\mathrm{AKT} / \mathrm{mTOR} / \mathrm{AMPK} / \mathrm{ULK} 1$ signaling pathway in astrocytes. Further and most notably, we reveal that NR and metformin rescued mitophagy defect in the POLG-mutant astrocytes, providing new possibilities for the treatment of these disorders by using mitophagy-enhancing reagents.

\section{DATA AVAILABILITY STATEMENT}

The datasets presented in this study can be found in online repositories. The names of the repository/repositories and accession number(s) can be found in the article/ Supplementary Material.

\section{ETHICS STATEMENT}

The project was approved by the Western Norway Committee for Ethics in Health Research (REK nr. 2012/919). The patients/participants provided their written informed consent to participate in this study.

\section{REFERENCES}

Acosta, C., Anderson, H., and Anderson, C. (2017). Astrocyte dysfunction in Alzheimer disease. J. Neurosci. Res. 95, 2430-2447. doi: 10.1002/jnr.24075

Bhansali, S., Bhansali, A., and Dhawan, V. (2020). Metformin promotes mitophagy in mononuclear cells: a potential in vitro model for unraveling metformin's mechanism of action. Ann. N. Y. Acad. Sci. 1463, 23-36. doi: 10.1111/nyas. 14141

Bharath, L. P., Agrawal, M., McCambridge, G., Nicholas, D. A., Hasturk, H., Liu, J., et al. (2020). Metformin enhances autophagy

\section{AUTHOR CONTRIBUTIONS}

$\mathrm{KL}$ and $\mathrm{LB}$ contributed to the conceptualization. $\mathrm{AC}$ and $\mathrm{KL}$ contributed to the methodology. AC, KL, CK, YH, and $\mathrm{AK}$ contributed to the investigation. $\mathrm{AC}, \mathrm{KL}$, and $\mathrm{LB}$ contributed to writing the original draft. AC, KL, CK, YH, AK, EF, GS, JW, XL, and $\mathrm{LB}$ contributed to the writing - review and editing. LB, GS, $\mathrm{EF}$, and $\mathrm{AC}$ contributed to the funding acquisition. KL, GS, and $\mathrm{LB}$ contributed to the resources. KL, LB, and XL contributed to the supervision. All authors agreed to the authorships.

\section{FUNDING}

This work was supported by funding from the Norwegian Research Council (project number: 229652), Rakel og Otto Kr.Bruuns legat. GS was partly supported by the Norwegian Research Council through its Centres of Excellence funding scheme (project number: 262613). AC was supported by the Natural Science Foundation of China (81972351), the National "111" Project (B20058), the Special Foundation for Taishan Scholars (ts20110814 and tshw201502056), the Department of Science and Technology of Shandong Province (2020CXGC010903 and ZR2019ZD33), the Clinical Research Center of Shandong University (2020SDUCRCB002), the Jinan Science and Technology Bureau of Shandong Province (2019GXRC006), and the China Scholarship Council (project number: 201906220275). EF was supported by HELSE SØRØST (project number: 2017056 and 2020001), the Research Council of Norway (project number: 262175 and 277813), the National Natural Science Foundation of China (project number: 81971327), and Akershus University Hospital Strategic grant (project number: 269901).

\section{ACKNOWLEDGMENTS}

The authors encourage all lab members for discussions and critical reading of the manuscript. The authors grateful to the Molecular Imaging Centre, Flow Cytometry Core Facility at the University of Bergen in Norway.

\section{SUPPLEMENTARY MATERIAL}

The Supplementary Material for this article can be found online at: https://www.frontiersin.org/articles/10.3389/fcell.2021. 737304/full\#supplementary-material

and normalizes mitochondrial function to alleviate aging-associated inflammation. Cell Metab. 32, 44-55.e6. doi: 10.1016/j.cmet.2020. 04.015

Burté, F., Carelli, V., Chinnery, P. F., and Yu-Wai-Man, P. (2015). Disturbed mitochondrial dynamics and neurodegenerative disorders. Nat. Rev. Neurol. 11, 11-24. doi: 10.1038/nrneurol.2014.228

Cea, M., Cagnetta, A., Fulciniti, M., Tai, Y.-T., Hideshima, T., Chauhan, D., et al. (2012). Targeting NAD+ salvage pathway induces autophagy in multiple myeloma cells via mTORC1 and extracellular signal-regulated kinase (ERK1/2) inhibition. Blood 120, 3519-3529. doi: 10.1182/blood-2012-03-416776 
Deas, E., Wood, N. W., and Plun-Favreau, H. (2011). Mitophagy and Parkinson's disease: the PINK1-parkin link. Biochim. Biophys. Acta 1813, 623-633. doi: 10.1016/j.bbamcr.2010.08.007

EauClaire, S. F., and Webb, C. J. (2019). A CRISPR/Cas9 method to generate heterozygous alleles in Saccharomyces cerevisiae. Yeast 36, 607-615. doi: 10. 1002/yea.3432

Egan, D. F., Shackelford, D. B., Mihaylova, M. M., Gelino, S., Kohnz, R. A., Mair, W., et al. (2011). Phosphorylation of ULK1 (hATG1) by AMP-activated protein kinase connects energy sensing to mitophagy. Science 331, 456-461.

Fang, E. F., Hou, Y., Lautrup, S., Jensen, M. B., Yang, B., SenGupta, T., et al. (2019a). NAD augmentation restores mitophagy and limits accelerated aging in Werner syndrome. Nat. Commun. 10:5284. doi: 10.1038/s41467-019-13172-8

Fang, E. F., Hou, Y., Palikaras, K., Adriaanse, B. A., Kerr, J. S., Yang, B., et al. (2019b). Mitophagy inhibits amyloid- $\beta$ and tau pathology and reverses cognitive deficits in models of Alzheimer's disease. Nat. Neurosci. 22, 401-412. doi: 10.1038/s41593-018-0332-9

Fang, E. F., Lautrup, S., Hou, Y., Demarest, T. G., Croteau, D. L., Mattson, M. P., et al. (2017). NAD(+) in aging: molecular mechanisms and translational implications. Trends Mol. Med. 23, 899-916. doi: 10.1016/j.molmed.2017.08. 001

Fang, E. F., Scheibye-Knudsen, M., Brace, L. E., Kassahun, H., SenGupta, T., Nilsen, H., et al. (2014). Defective mitophagy in XPA via PARP-1 hyperactivation and NAD+/SIRT1 reduction. Cell 157, 882-896. doi: 10.1016/j.cell.2014.03.026

Fang, E. F., Scheibye-Knudsen, M., Chua, K. F., Mattson, M. P., Croteau, D. L., and Bohr, V. A. (2016). Nuclear DNA damage signalling to mitochondria in ageing. Nat. Rev. Mol. Cell Biol. 17, 308-321. doi: 10.1038/nrm.2016.14

Fivenson, E. M., Lautrup, S., Sun, N., Scheibye-Knudsen, M., Stevnsner, T., Nilsen, H., et al. (2017). Mitophagy in neurodegeneration and aging. Neurochem. Int. 109, 202-209. doi: 10.1016/j.neuint.2017.02.007

Graziewicz, M. A., Longley, M. J., and Copeland, W. C. (2006). DNA polymerase gamma in mitochondrial DNA replication and repair. Chem. Rev. 106, 383-405. doi: $10.1021 / \mathrm{cr} 040463 \mathrm{~d}$

Guerra, F., Girolimetti, G., Beli, R., Mitruccio, M., Pacelli, C., Ferretta, A., et al. (2019). Synergistic effect of mitochondrial and lysosomal dysfunction in Parkinson's disease. Cells 8:452. doi: 10.3390/cells8050452

Haydon, P. G., and Carmignoto, G. (2006). Astrocyte control of synaptic transmission and neurovascular coupling. Physiol. Rev. 86, 1009-1031. doi: 10.1152/physrev.00049.2005

Heras-Sandoval, D., Pérez-Rojas, J. M., Hernández-Damián, J., and PedrazaChaverri, J. (2014). The role of PI3K/AKT/mTOR pathway in the modulation of autophagy and the clearance of protein aggregates in neurodegeneration. Cell. Signal. 26, 2694-2701. doi: 10.1016/j.cellsig.2014.08.019

Hikmat, O., Naess, K., Engvall, M., Klingenberg, C., Rasmussen, M., Tallaksen, C. M., et al. (2020). Simplifying the clinical classification of polymerase gamma (POLG) disease based on age of onset; studies using a cohort of 155 cases. J. Inherit. Metab. Dis. 43, 726-736. doi: 10.1002/jimd.12211

Homma, T., Ishibashi, D., Nakagaki, T., Satoh, K., Sano, K., Atarashi, R., et al. (2014). Increased expression of p62/SQSTM1 in prion diseases and its association with pathogenic prion protein. Sci. Rep. 4, 1-7.

Hung, C.-M., Lombardo, P. S., Malik, N., Brun, S. N., Hellberg, K., Van Nostrand, J. L., et al. (2021). AMPK/ULK1-mediated phosphorylation of Parkin ACT domain mediates an early step in mitophagy. Sci. Adv. 7, eabg4544. doi: 10. 1126/sciadv.abg4544

Ignatenko, O., Chilov, D., Paetau, I., de Miguel, E., Jackson, C. B., Capin, G., et al. (2018). Loss of mtDNA activates astrocytes and leads to spongiotic encephalopathy. Nat. Commun. 9:70. doi: 10.1038/s41467-017-01859-9

Jackson, J. G., and Robinson, M. B. (2018). Regulation of mitochondrial dynamics in astrocytes: mechanisms, consequences, and unknowns. Glia 66, 1213-1234. doi: 10.1002/glia.23252

Kerr, J. S., Adriaanse, B. A., Greig, N. H., Mattson, M. P., Cader, M. Z., Bohr, V. A., et al. (2017). Mitophagy and Alzheimer's disease: cellular and molecular mechanisms. Trends Neurosci. 40, 151-166. doi: 10.1016/j.tins.2017.01.002

Kim, J., Kundu, M., Viollet, B., and Guan, K.-L. (2011). AMPK and mTOR regulate autophagy through direct phosphorylation of Ulk1. Nat. Cell Biol. 13, 132-141. doi: $10.1038 /$ ncb 2152

Kishton, R. J., Barnes, C. E., Nichols, A. G., Cohen, S., Gerriets, V. A., Siska, P. J., et al. (2016). AMPK is essential to balance glycolysis and mitochondrial metabolism to control T-ALL cell stress and survival. Cell Metab. 23, 649-662. doi: 10.1016/j.cmet.2016.03.008
Kodali, M., Attaluri, S., Madhu, L. N., Shuai, B., Upadhya, R., Gonzalez, J. J., et al. (2021). Metformin treatment in late middle age improves cognitive function with alleviation of microglial activation and enhancement of autophagy in the hippocampus. Aging Cell 20:e13277. doi: 10.1111/acel.13277

Lautrup, S., Lou, G., Aman, Y., Nilsen, H., Tao, J., and Fang, E. F. (2019). Microglial mitophagy mitigates neuroinflammation in Alzheimer's disease. Neurochem. Int. 129:104469. doi: 10.1016/j.neuint.2019.104469

Lazarou, M., Sliter, D. A., Kane, L. A., Sarraf, S. A., Wang, C., Burman, J. L., et al. (2015). The ubiquitin kinase PINK1 recruits autophagy receptors to induce mitophagy. Nature 524, 309-314. doi: 10.1038/nature14893

Lee, S., Zhang, C., and Liu, X. (2015). Role of glucose metabolism and ATP in maintaining PINK1 levels during Parkin-mediated mitochondrial damage responses. J. Biol. Chem. 290, 904-917. doi: 10.1074/jbc.M114.606798

Liang, K. X., Kianian, A., Chen, A., Kristiansen, C. K., Hong, Y., Furriol, J., et al. (2020a). Stem cell derived astrocytes with POLG mutations and mitochondrial dysfunction including abnormal NAD+ metabolism is toxic for neurons. Biorxiv[Preprint] doi: 10.1101/2020.12.20.423652

Liang, K. X., Kristiansen, C. K., Mostafavi, S., Vatne, G. H., Zantingh, G. A., Kianian, A., et al. (2020b). Disease-specific phenotypes in iPSC-derived neural stem cells with POLG mutations. EMBO Mol. Med. 12:e12146. doi: 10.15252/ emmm.202012146

Liang, K. X., Vatne, G. H., Kristiansen, C. K., Ievglevskyi, O., Kondratskaya, E., Glover, J. C., et al. (2021). N-acetylcysteine amide ameliorates mitochondrial dysfunction and reduces oxidative stress in hiPSC-derived dopaminergic neurons with POLG mutation. Exp. Neurol. 337:113536. doi: 10.1016/j. expneurol.2020.113536

Liddelow, S. A., Guttenplan, K. A., Clarke, L. E., Bennett, F. C., Bohlen, C. J., Schirmer, L., et al. (2017). Neurotoxic reactive astrocytes are induced by activated microglia. Nature 541, 481-487. doi: 10.1038/nature21029

Lin, M. T., and Beal, M. F. (2006). Mitochondrial dysfunction and oxidative stress in neurodegenerative diseases. Nature 443, 787-795. doi: 10.1038/nature05292

Liu, X., Liao, X., Rao, X., Wang, B., Zhang, J., Xu, G., et al. (2020). The lysosomal membrane protein LAMP-2 is dispensable for PINK1/Parkinmediated mitophagy. FEBS Lett. 594, 823-840. doi: 10.1002/1873-3468.13663

Ma, S., Attarwala, I. Y., and Xie, X.-Q. (2019). SQSTM1/p62: a potential target for neurodegenerative disease. ACS Chem. Neurosci. 10, 2094-2114. doi: 10.1021/ acschemneuro.8b00516

Maldonado, P. D., Chánez-Cárdenas, M. E., and Fernández-López, A. (2018). Mechanisms of cell damage in neurological diseases and putative neuroprotective strategies. Oxid. Med. Cell. Longev. 2018:9784319. doi: $10.1155 / 2018 / 9784319$

Manning, B. D., and Toker, A. (2017). AKT/PKB Signaling: navigating the network. Cell 169, 381-405. doi: 10.1016/j.cell.2017.04.001

Mattson, M. P., Gleichmann, M., and Cheng, A. (2008). Mitochondria in neuroplasticity and neurological disorders. Neuron 60, 748-766. doi: 10.1016/j. neuron.2008.10.010

Mei, R., Lou, P., You, G., Jiang, T., Yu, X., and Guo, L. (2020). 17 $\beta$-Estradiol induces mitophagy upregulation to protect chondrocytes the SIRT1-mediated AMPK/mTOR signaling pathway. Front. Endocrinol. 11:615250. doi: 10.3389/ fendo.2020.615250

Ng, C.-H., Guan, M. S. H., Koh, C., Ouyang, X., Yu, F., Tan, E.-K., et al. (2012). AMP kinase activation mitigates dopaminergic dysfunction and mitochondrial abnormalities in Drosophila models of Parkinson's disease. J. Neurosci. 32, 14311-14317. doi: 10.1523/JNEUROSCI.0499-12.2012

Owen, M. R., Doran, E., and Halestrap, A. P. (2000). Evidence that metformin exerts its anti-diabetic effects through inhibition of complex 1 of the mitochondrial respiratory chain. Biochem. J. 348, 607-614. doi: 10.1042/02646021:3480607

Perluigi, M., Di Domenico, F., and Butterfield, D. A. (2015). mTOR signaling in aging and neurodegeneration: at the crossroad between metabolism dysfunction and impairment of autophagy. Neurobiol. Dis. 84, 39-49. doi: 10. 1016/j.nbd.2015.03.014

Plotegher, N., and Duchen, M. R. (2017). Mitochondrial dysfunction and neurodegeneration in lysosomal storage disorders. Trends Mol. Med. 23, 116134. doi: 10.1016/j.molmed.2016.12.003

Price, N. L., Gomes, A. P., Ling, A. J. Y., Duarte, F. V., Martin-Montalvo, A., North, B. J., et al. (2012). SIRT1 is required for AMPK activation and the beneficial effects of resveratrol on mitochondrial function. Cell Metab. 15, 675-690. doi: 10.1016/j.cmet.2012.04.003 
Richetin, K., Steullet, P., Pachoud, M., Perbet, R., Parietti, E., Maheswaran, M., et al. (2020). Tau accumulation in astrocytes of the dentate gyrus induces neuronal dysfunction and memory deficits in Alzheimer's disease. Nat. Neurosci. 23, 1567-1579. doi: 10.1038/s41593-020-00728-x

Saxton, R. A., and Sabatini, D. M. (2017). mTOR Signaling in growth, metabolism, and disease. Cell 168, 960-976. doi: 10.1016/j.cell.2017.02.004

Sheng, Z.-H., and Cai, Q. (2012). Mitochondrial transport in neurons: impact on synaptic homeostasis and neurodegeneration. Nat. Rev. Neurosci. 13, 77-93. doi: $10.1038 / \mathrm{nrn} 3156$

Song, P., Li, S., Wu, H., Gao, R., Rao, G., Wang, D., et al. (2016). Parkin promotes proteasomal degradation of $\mathrm{p} 62$ : implication of selective vulnerability of neuronal cells in the pathogenesis of Parkinson's disease. Protein Cell 7, 114-129. doi: 10.1007/s13238-015-0230-9

Sonninen, T.-M., Hämäläinen, R. H., Koskuvi, M., Oksanen, M., Shakirzyanova, A., Wojciechowski, S., et al. (2020). Metabolic alterations in Parkinson's disease astrocytes. Sci. Rep. 10:14474. doi: 10.1038/s41598-020-71329-8

Tzoulis, C., Tran, G. T., Coxhead, J., Bertelsen, B., Lilleng, P. K., Balafkan, N., et al. (2014). Molecular pathogenesis of polymerase gammarelated neurodegeneration. Ann. Neurol. 76, 66-81. doi: 10.1002/ana. 24185

Vannini, N., Campos, V., Girotra, M., Trachsel, V., Rojas-Sutterlin, S., Tratwal, J., et al. (2019). The NAD-booster nicotinamide riboside potently stimulates hematopoiesis through increased mitochondrial clearance. Cell Stem Cell 24, 405.e-418.e. doi: 10.1016/j.stem.2019. 02.012

Vives-Bauza, C., Zhou, C., Huang, Y., Cui, M., de Vries, R. L. A., Kim, J., et al. (2010). PINK1-dependent recruitment of Parkin to mitochondria in mitophagy. Proc. Natl. Acad. Sci. U. S. A. 107, 378-383. doi: 10.1073/pnas.0911187107

Wang, J.-L., and Xu, C.-J. (2020). Astrocytes autophagy in aging and neurodegenerative disorders. Biomed. Pharmacother. 122:109691. doi: 10.1016/ j.biopha.2019.109691
Wang, Y., An, H., Liu, T., Qin, C., Sesaki, H., Guo, S., et al. (2019). Metformin improves mitochondrial respiratory activity through activation of AMPK. Cell Rep. 29, 1511-1523.e5.

Youle, R. J., and Narendra, D. P. (2011). Mechanisms of mitophagy. Nat. Rev. Mol. Cell Biol. 12, 9-14. doi: 10.1038/nrm3028

Zheng, D., Zhang, Y., Zheng, M., Cao, T., Wang, G., Zhang, L., et al. (2019). Nicotinamide riboside promotes autolysosome clearance in preventing doxorubicin-induced cardiotoxicity. Clin. Sci. (Lond.). 133, 1505-1521. doi: $10.1042 /$ CS20181022

Conflict of Interest: EF has a CRADA arrangement with ChromaDex and is a consultant to Aladdin Healthcare Technologies and the Vancouver Dementia Prevention Centre.

The remaining authors declare that the research was conducted in the absence of any commercial or financial relationships that could be construed as a potential conflict of interest.

Publisher's Note: All claims expressed in this article are solely those of the authors and do not necessarily represent those of their affiliated organizations, or those of the publisher, the editors and the reviewers. Any product that may be evaluated in this article, or claim that may be made by its manufacturer, is not guaranteed or endorsed by the publisher.

Copyright (๑) 2021 Chen, Kristiansen, Hong, Kianian, Fang, Sullivan, Wang, Li, Bindoff and Liang. This is an open-access article distributed under the terms of the Creative Commons Attribution License (CC BY). The use, distribution or reproduction in other forums is permitted, provided the original author(s) and the copyright owner(s) are credited and that the original publication in this journal is cited, in accordance with accepted academic practice. No use, distribution or reproduction is permitted which does not comply with these terms. 